\title{
Practical aspects of a pulse generator calibration
}

\author{
Martin Hudlička ${ }^{1}$, mhudlicka@cmi.cz, Marco A. Azpúrua ${ }^{2}$, Murat Celep $^{3}$ \\ ${ }^{1}$ Czech metrology institute, Brno, Czech Republic \\ ${ }^{2}$ Universitat Politècnica de Catalunya, Barcelona, Spain \\ ${ }^{3}$ TUBITAK Ulusal Metroloji Enstitüsü (UME), Gebze, Turkey
}

Measuring receivers are used for measurement of radio disturbance in the frequency range typically $9 \mathrm{kHz}$ to $18 \mathrm{GHz}$. Such receivers can be either electromagnetic interference (EMI) receivers or spectrum analyzers with the quasi-peak (QP) detector [1], [2]. Requirements for measuring receivers are discussed in European CISPR documents (Comité International Spécial des Perturbations Rádioélectriques) and US standards ANSI 63.2 (QP parts derived from CISPR). Although novel techniques are being evaluated [3], the traditional way of checking compliance of the receiver with requirements of these standards is using a calibrated pulse generator [4]. The calibration of pulse generators is discussed in the standard EN 550161-1 [5], which is the harmonized version of the international standard IEC/CISPR 16-1-1 (currently Ed. 4 [6]). In the standard [5], however, only a very brief description of the methods is given and technical details are hidden. The measurement uncertainty of the pulse generator characterization is not discussed in the standards, however, it was discussed in several previous works [7], [8]. This paper aims to provide a more thorough description of particular calibration methods together with practical hints which may be useful for students, calibration engineers and practitioners.

\section{Pulse generators}

According to [5], a pulse generator is an instrument capable of generating time-domain rectangular pulses, or a pulse-modulated RF signal. Rectangular pulses are typically used for lower frequencies (bands A/B), pulse-modulated RF signals for higher frequencies (bands $\mathrm{C} / \mathrm{D})$ because of the risk of receiver damage due to high peak voltages. The base-band pulse generators usually comprise of an energy-storage device (electrostatic, magnetic field) and a switch which discharges a fraction or all of the energy into a load. The pulse-modulated RF generator uses a harmonic signal with a pulse envelope. The spectrum is similar to a rectangular pulse (upconverted to the carrier frequency $f_{c}$ ), maximum of the spectrum is at $f_{c}$. The spectrum is uniform in a given bandwidth, which implies that pulses with longer duration can be used with lower amplitudes compared to base-band pulse generators (lower risk of 
measuring receiver damage). The typical spectrum of a rectangular base-band pulse and a pulse-modulated RF signal is shown in Fig. 1.
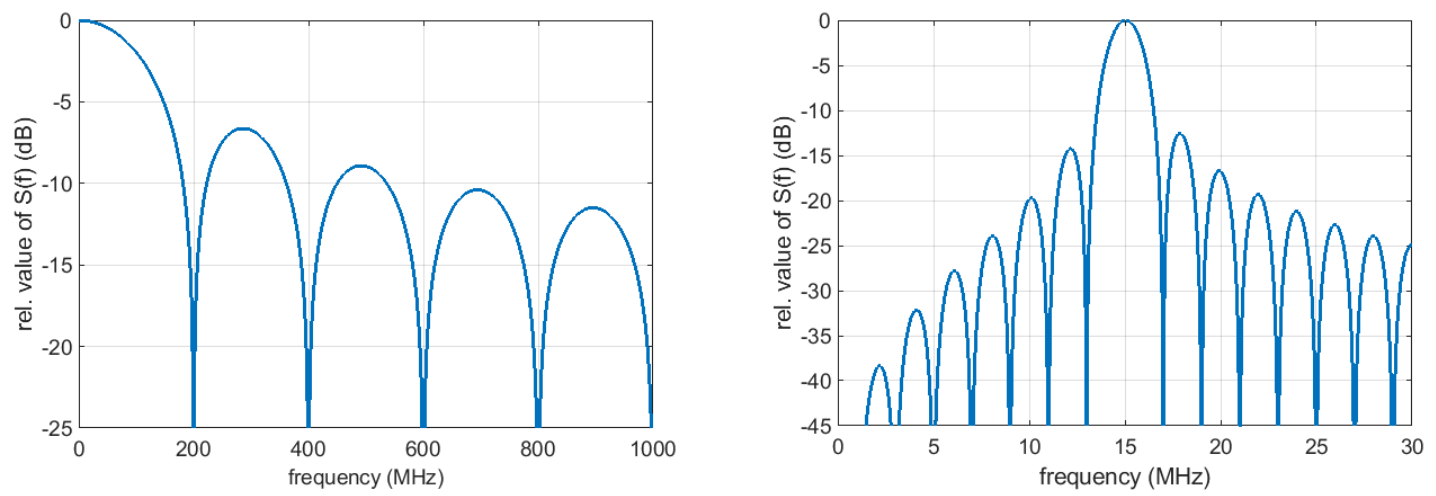

Fig. 1 Typical spectrum of a rectangular base-band pulse (left) and pulse-modulated RF signal (right).

The terminology used with the pulse generator characterization is summarized in Tab. 1 . All quantities for characterization of pulse generators have dimensional unit $[\mathrm{V} / \mathrm{Hz}]$ or its mathematical equivalent.

\begin{tabular}{|c|c|c|}
\hline Quantity & Definition & Notes \\
\hline $\begin{array}{l}\text { Spectrum } \\
\text { amplitude }\end{array}$ & $S(f)=2|V(f)|$ & $\begin{array}{l}V(f) \text { is the Fourier transform of the } v(t) \text { signal in } \\
\text { the time-domain [9], [10]. It is the measure of the } \\
\text { amplitude spectrum at a certain frequency, } \\
\text { expressed in }[\mathrm{dB} \mu \mathrm{V} / \mathrm{MHz}] \text {. For a rectangular } \\
\text { pulse with amplitude } A \text { and duration } T \text { and low } \\
\text { frequencies }(f<<T) \text {, we can write } S(f)=2 A T \text {. }\end{array}$ \\
\hline Impulse area & $A_{i m p}=\int_{0}^{T} v(t) d t$ & $\begin{array}{l}\text { Voltage-time area of a pulse defined by the } \\
\text { integral. Sometimes referred to as impulse } \\
\text { strength, typically expressed in }[\mu \mathrm{Vs}] \text { or } \\
{[\mathrm{dB} \mu \mathrm{Vs}] \text {. Related to spectral density. } T \text { is one }} \\
\text { pulse period. }\end{array}$ \\
\hline Spectral intensity & $S(f)=2 A_{i m p}$ & This definition can be found in [5] and [10]. \\
\hline Spectral density & $D=\sqrt{2} \times 10^{6} A_{i m p}$ & $\begin{array}{l}\text { Defined in }[5] \text {. Alternative term "impulse } \\
\text { strength" can be found. The term } \sqrt{ } 2 \text { stands for } \\
\text { the peak to RMS conversion. } D \text { is calculated in } \\
{[\mu \mathrm{V} / \mathrm{MHz}] \text { for } A_{\text {imp }} \text { given in }[\mu \mathrm{Vs}] \text {. }}\end{array}$ \\
\hline
\end{tabular}

Tab. 1 Quantities used for characterization of pulse generators. 


\section{Calibration methods}

The various pulse generator calibration methods will be demonstrated on the example calibration of the CISPR pulse generator IGUU2916 (base-band pulse generator, manufacturer Schwarzbeck, Germany). The generator is shown in Fig. 2. The methods are finally compared with regards to the measurement uncertainty, feasibility and required instrumentation. The polarity was always $(+)$ and the amplitude of the main generator (mechanical relay contact) was always $60 \mathrm{~dB} \mu \mathrm{V}$, the amplitude of the auxiliary (semiconductor switch) generator was $40 \mathrm{~dB} \mu \mathrm{V}$ and the pulse repetition rate was changed according to the band.

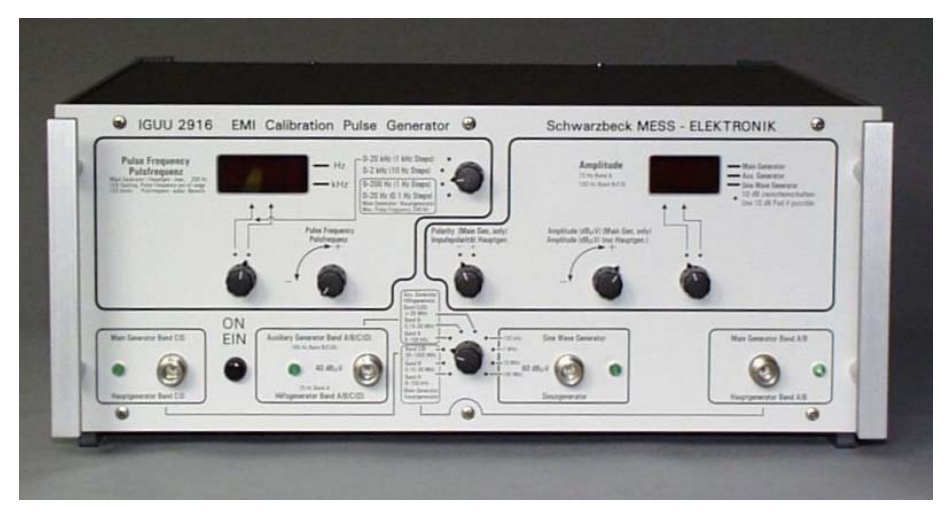

Fig. 2 CISPR pulse generator Schwarzbeck IGUU 2916.

In the EN 55016-1-1 [5] (CISPR 16-1-1 [6]) there are specified impulse areas of a typical generator for both open-circuit and $50 \Omega$ load, see Tab. 2. The values shown in this paper correspond to the measurement of the spectrum amplitude (calculated from the impulse area) into $50 \Omega$ nominal load.

\begin{tabular}{|l|c|c|c|c|}
\hline \multicolumn{1}{|c|}{ Band } & A & B & C & D \\
\hline $\begin{array}{l}\text { Frequency } \\
\text { range }\end{array}$ & $\begin{array}{c}\{9-150\} \\
\mathrm{kHz}\end{array}$ & $\{0.15-30\} \mathrm{MHz}$ & $\{30-300\} \mathrm{MHz}$ & $\{300-1000\} \mathrm{MHz}$ \\
\hline $\begin{array}{l}\text { Impulse area } \\
\text { (open-circuit) }\end{array}$ & $13.5 \mu \mathrm{Vs}$ & $0.316 \mu \mathrm{Vs}$ & $0.044 \mu \mathrm{Vs}$ & $0.044 \mu \mathrm{Vs}$ \\
\hline $\begin{array}{l}\text { Impulse area } \\
\text { (into } 50 \Omega \\
\text { load) }\end{array}$ & $6.75 \mu \mathrm{Vs}$ & $0.158 \mu \mathrm{Vs}$ & $0.022 \mu \mathrm{Vs}$ & $0.022 \mu \mathrm{Vs}$ \\
\hline Repetition rate & $25 \mathrm{~Hz}$ & $100 \mathrm{~Hz}$ & $100 \mathrm{~Hz}$ & $100 \mathrm{~Hz}$ \\
\hline
\end{tabular}


Tab. 2 Impulse area of a generator for different bands specified in the EN 55016-1-1

standard.

\section{Fourier transform of a time-domain pulse waveform}

The spectrum amplitude is determined by direct acquisition of the pulse generator output voltage using an oscilloscope and conversion into the frequency domain. For this purpose, a digital real-time oscilloscope (DRTO) or an equivalent-time sampling oscilloscope (DSO) can be used. The method is useful for base-band pulse generators, it is simple and time-efficient. Corrections for the cable (attenuator) properties and oscilloscope transfer function must be performed. A DRTO triggers directly the measured pulse. The traceability of DRTO is complicated due to the nonlinear behaviour of modern analogue-to-digital converters and the transfer function correction. A DSO needs an external trigger signal, which is usually derived from the measured signal itself (approx. $20 \mathrm{~ns}$ delay line is used). The measurement is traceable to the electro-optic sampling system. A general diagram of the measurement setup using the DRTO and DSO is shown in Fig. 3.
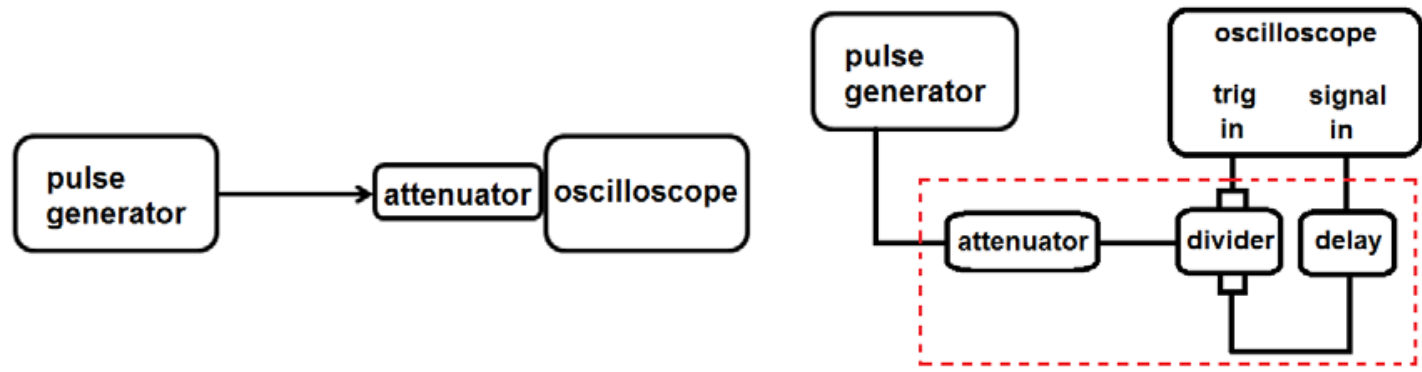

Fig. 3 Typical measurement setup with the use of a DRTO (left) and DSO (right).

The measurement equation is the following:

$$
S(f)=20 \log \left(\frac{|V(f)| \sqrt{2} \cdot 10^{6} \cdot k_{\text {ATT }} \cdot k_{\text {osc }}}{N_{F F T} \Delta f / 10^{6}}\right) \quad[\mathrm{dB} \mu \mathrm{V} / \mathrm{MHz}],
$$

where $V(f)$ is the Fourier transform of the voltage trace from oscilloscope in [V], $N_{F F T}$ is the FFT length, $\Delta f$ is the frequency resolution in $[\mathrm{Hz}], k_{A T T}$ is the total attenuation of the signal path, that is, the cables and external attenuators connected between the generator and oscilloscope, $k_{o s c}$ is a factor taking into account the oscilloscope frequency response. A cable with attenuators on both sides should be used in order to reduce the pulse amplitude and improve the mismatch uncertainty. 
Following text shows an example of measured and calculated results. The spectrum amplitude was calculated using (1) from oscilloscope voltage samples corrected for the cable and attenuator and oscilloscope transfer function. Following uncertainty contributions apply:

- impedance mismatch correction between device 1 and 2 (e.g. between a generator and cable) $u_{M}=20 \log \left(1+\left|\Gamma_{1}\right|\left|\Gamma_{2}\right|\right) \quad(\mathrm{dB})$, where $\left|\Gamma_{1}\right|$ and $\left|\Gamma_{2}\right|$ are magnitudes of reflection coefficients of devices 1 and 2 (in linear scale), respectively,

- type A uncertainty was calculated from repeated calculations of the spectrum amplitude for all captured time traces, its value was determined from $n$ measurements in a standard way as

$$
u_{A y}=\sqrt{\frac{1}{n(n-1)} \sum_{i=1}^{n}\left(y_{i}-\bar{y}\right)} .
$$

The output reflection coefficient of the IGUU 2916 Main generator is not specified in the datasheet [11] and it was calculated from a hypothetical generator composed of an ideal voltage source with an inner resistance $R_{\text {in }}$ by measuring the output voltage (peak maximum) of the main generator into two different load impedances $50 \Omega$ and $1000 \Omega$, respectively.

The measured results of the Fourier transform method with measurement uncertainty are summarized in Tab. 4. The example of measurement uncertainty evaluation for the Band A, main generator $(60 \mathrm{~dB} \mu \mathrm{V})$, is given in Tab. 3 .

\begin{tabular}{|c|c|c|c|c|c|c|c|}
\hline $\begin{array}{c}\text { Quantity } \\
\qquad X_{i}\end{array}$ & $\begin{array}{c}\text { Estimate } \\
x_{i}\end{array}$ & $\begin{array}{l}\text { Estimate } \\
\text { uncertainty } \\
u\left(x_{i}\right)\end{array}$ & $\begin{array}{l}\text { Probability } \\
\text { distribution }\end{array}$ & Divisor & $\begin{array}{c}\text { Standard } \\
\text { uncertainty } \\
u\left(x_{i}\right)\end{array}$ & $\begin{array}{c}\text { Sensitivity } \\
\text { coefficient } \\
c_{i}\end{array}$ & $\begin{array}{c}\text { Uncertainty } \\
\text { contribution } \\
u\left(y_{i}\right)\end{array}$ \\
\hline$k_{\text {osc }}$ & $0 \mathrm{~dB}$ & $0.060 \mathrm{~dB}$ & normal & 1 & $0.060 \mathrm{~dB}$ & 1 & $0.060 \mathrm{~dB}$ \\
\hline$k_{A T T}$ & $0 \mathrm{~dB}$ & $0.020 \mathrm{~dB}$ & normal & 1 & $0.020 \mathrm{~dB}$ & 1 & $0.020 \mathrm{~dB}$ \\
\hline$M_{\text {ATT-osc }}$ & $0 \mathrm{~dB}$ & $0.008 \mathrm{~dB}$ & U-shaped & 1.41 & $0.006 \mathrm{~dB}$ & 1 & $0.006 \mathrm{~dB}$ \\
\hline$M_{\text {gen-ATT }}$ & $0 \mathrm{~dB}$ & $0.001 \mathrm{~dB}$ & U-shaped & 1.41 & $0.0007 \mathrm{~dB}$ & 1 & $0.0007 \mathrm{~dB}$ \\
\hline type A & $0 \mathrm{~dB}$ & $0.038 \mathrm{~dB}$ & rectangular & 1.73 & $0.022 \mathrm{~dB}$ & 1 & $0.022 \mathrm{~dB}$ \\
\hline & & & & & & total & $0.067 \mathrm{~dB}$ \\
\hline & & & & & & $\begin{array}{l}\text { expanded } \\
\text { uncertainty } \\
\quad(k=2)\end{array}$ & $0.13 \mathrm{~dB}$ \\
\hline
\end{tabular}

Tab. 3 Example of uncertainty calculation, band A, $f_{\text {rep }}=25 \mathrm{~Hz}$, frequency $9 \mathrm{kHz}$. 


\section{Intermediate-frequency measurement method}

This method utilizes an EMI measuring receiver and its intermediate frequency output. It is referred to as "video pulse technique" in MIL-STD-462 [12], and it is referred to as "video pulse technique" and "area method" in [5]. The method uses a pulse signal and a reference CW signal (with known level) connected to a narrow-band filter, whereas the output of the filter (intermediate frequency) is acquired using an oscilloscope. The spectrum amplitude is then calculated from the response to both input signals at the frequency of the tuned filter (receiver) as follows

$$
S(f)=U_{r m s} / \mathrm{IBW},
$$

where $U_{r m s}(\mathrm{~V})$ is the level of $\mathrm{CW}$ signal which causes equal oscilloscope reading as the pulse signal, IBW $(\mathrm{Hz})$ is the impulse bandwidth of the used filter. The accuracy of the method is dependent on the accurate characterization of the receiver impulse bandwidth IBW [13]. The spectrum amplitude is calculated as the surface under the pulse envelope (i.e. positive amplitudes only), see Fig. 4. The measurement setup is shown in Fig. 5.

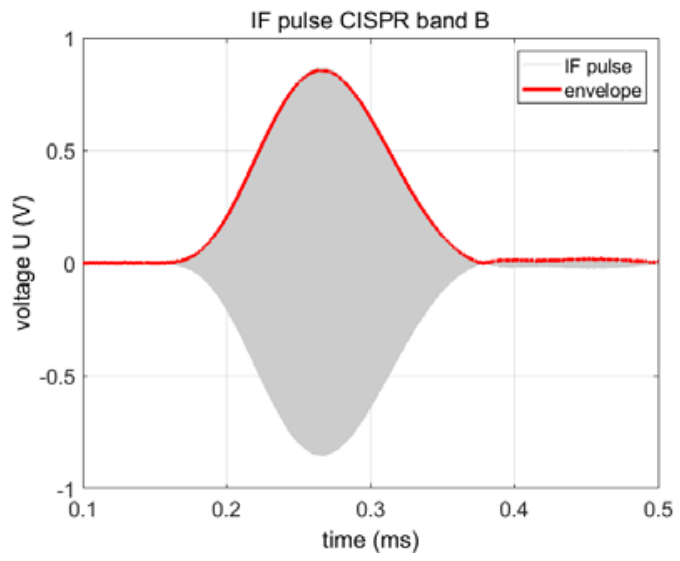

Fig. 4 Intermediate frequency measurement method. 


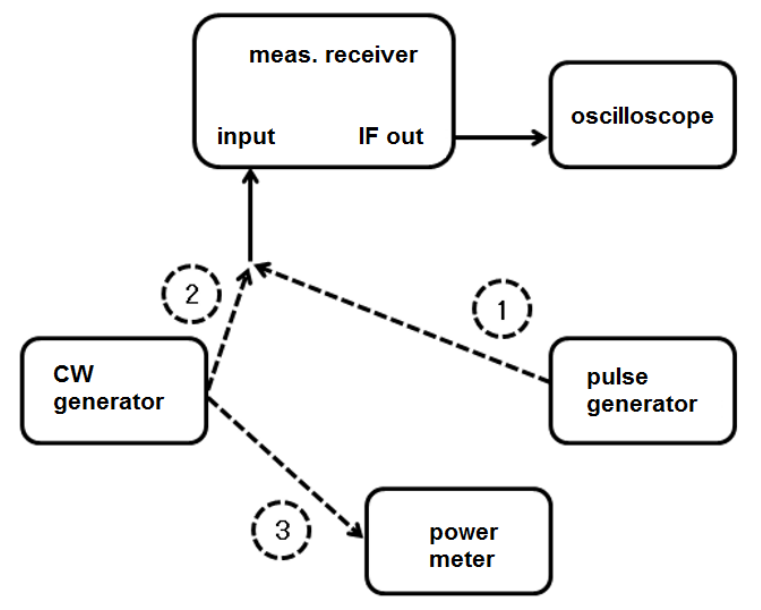

Fig. 5 Measurement setup of the intermediate frequency method.

The practical procedure is following: in the first step, the pulse generator output is connected to the receiver input and the response of the receiver's IF filter is captured using an oscilloscope (direct connection using a high-grade cable, without attenuators). The peak-topeak amplitude of the trace is measured as well. In the second step, a CW sine signal is connected to the receiver and its amplitude is changed until the oscilloscope peak-to-peak reading is the same as for the pulse signal. The RMS level of this sine signal is measured using a calibrated power meter. The attenuation of the cable from the generator to the receiver and from the receiver IF output to the oscilloscope is not important, as it cancels due to the ratio measurement. The measurement equation is following

$$
S(f)=20 \log \left(\frac{2 V_{p w m, r m s} V_{\text {env }}}{V_{\text {osc }, p p}} k_{\text {peak }} k_{\text {osc }} k_{I B W} I B W \cdot 10^{6}\right) \quad[\mathrm{dB} \mu \mathrm{V} / \mathrm{MHz}]
$$

where $V_{p w m, r m s}$ is the voltage across a $50 \Omega$ load of the $\mathrm{CW}$ sine signal calculated from the RMS power measured by the power meter in $[\mu \mathrm{V}]$

$V_{\text {env }}$ is the amplitude of the IF pulse envelope (see the example in Fig. 6) in [V]

$V_{o s c, p p}$ is the peak-to-peak amplitude of the receiver response (IF output) to a CW sine signal measured by the oscilloscope in [V]

$I B W$ is the receiver impulse bandwidth in $[\mathrm{Hz}]$

$k_{\text {peak }}$ takes into account the uncertainty of the peak ratio of the response to pulse/CW signal (dimensionless)

$k_{\text {osc }}$ takes into account the oscilloscope frequency response (dimensionless) 
$k_{I B W}$ takes into account the uncertainty of the determination of the impulse bandwidth (dimensionless).

The receiver impulse bandwidth is calculated as [13]

$$
I B W=\frac{V_{e n v}}{X \cdot \frac{10}{N} \sum_{k=1}^{N} v_{e n v, k}}[\mathrm{~Hz}],
$$

where $V_{\text {env }}$ is the amplitude of the IF pulse envelope (see the example in Fig. 6) in [V]

$X$ is the oscilloscope horizontal resolution in [s/div]

$N$ is the number of samples of the envelope [-]

$v_{\text {env,k }}$ represents the $k$-th sample of the IF pulse envelope in [V]

10 takes into account the number of oscilloscope horizontal screen divisions.
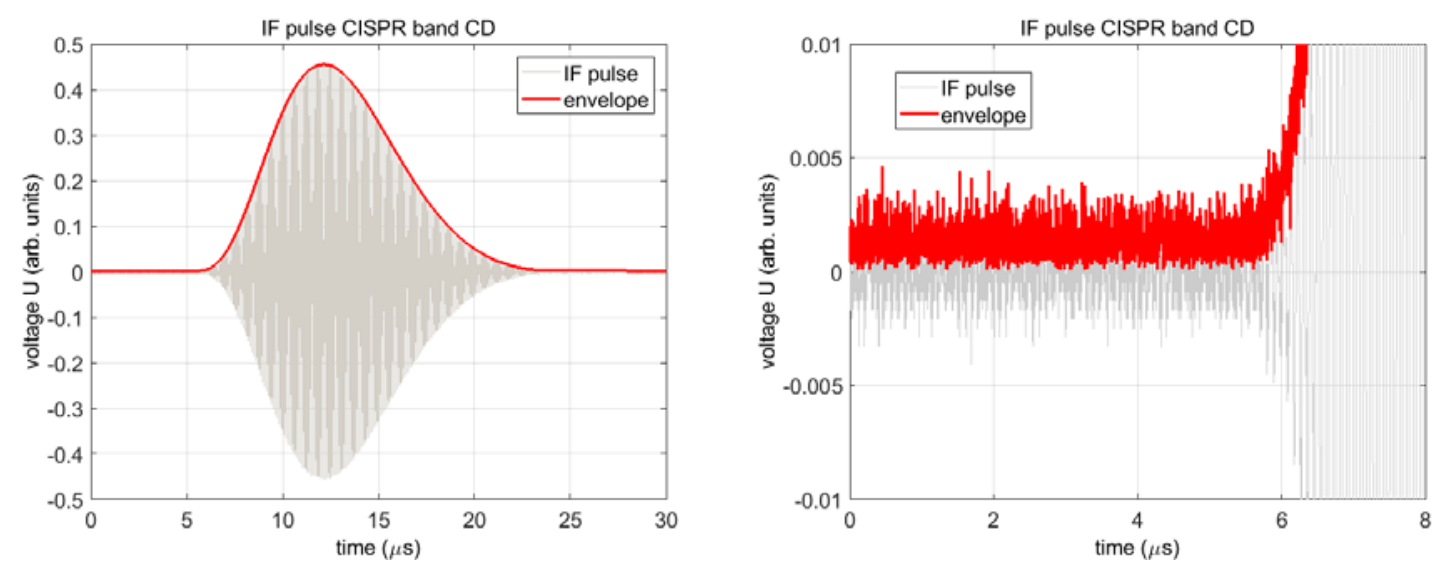

Fig. 6 Response of the receiver to a pulse signal + envelope of the signal (left), unfiltered envelope (right).

The determination of the IF pulse envelope can be done using different methods which results in slightly different calculated receiver impulse bandwidth and consequently spectrum amplitude. Either the envelope can be calculated as a moving average of the voltage IF trace (with e.g. 50 - 200 samples window), or it can be calculated as a magnitude of the Hilbert transform of the IF voltage trace (time-domain). It is convenient to filter the envelope trace using a low-pass filter in order to remove the noise (which is obvious in Fig. 6 right). The area under the envelope is then calculated as a sum of the voltage samples divided by the number of envelope samples. The spectrum amplitude was calculated using (6) from oscilloscope samples of the voltage at the IF output of an EMI receiver and their values together with the measurement uncertainty are given in Tab. 4. 


\section{Measurement of pulse amplitude and duration}

This method uses an oscilloscope, whereas the pulse is sampled with high time-resolution. The method is most suitable for pulse-modulated RF generators. The spectrum amplitude is calculated from the area of the pulse $[\mathrm{V} \cdot \mathrm{s}]$. The ideal measurement equation can be written as follows

$$
S(f)=U_{r m s} \cdot T \cdot k_{c o r},
$$

where $U_{r m s}$ is the un-modulated CW signal level, $T$ is the modulation pulse duration and $k_{c o r}$ is a correction factor taking into account the signal level change after switching ON the pulse. The pulse repetition rate should be stable and the correction factor should not change with $\mathrm{CW}$ frequency. An example of the pulse modulated $\mathrm{CW}$ signal with frequency $5 \mathrm{MHz}$ is shown in Fig. 7 (note the nonzero rising and trailing edge of the pulse due to the modulator non-idealities).

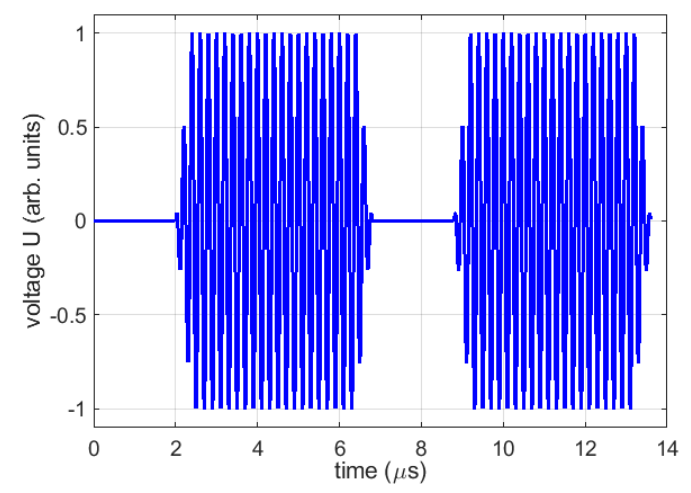

Fig. 7 Measurement of pulse amplitude and duration, pulse-modulated RF generator.

The method is also applicable for base-band pulse generators. The pulse shape must be very close to rectangular, which is not always true for the measured IGUU2916 main generator (especially band C/D). The pulse amplitude and duration is measured using an oscilloscope. The pulse shape in the time domain is generally distorted by the transfer function of the cable + attenuator(s) and the oscilloscope transfer function. In order to remove this effect correctly, one has to perform deconvolution of the transfer function in the frequency domain. The pulse shape, however, was acquired using a short oscilloscope epoch (only the pulse visible on the screen), which makes the frequency resolution very coarse and the correction is not easily applicable.

$$
S(f)=20 \log \left(\sqrt{2} A \cdot k_{\text {ATT }} \cdot \Delta T \cdot\left|\frac{\sin (\pi f T)}{\pi f T}\right| \cdot 10^{6}\right) \quad[\mathrm{dB} \mu \mathrm{V} / \mathrm{MHz}],
$$


where $A$ is the sum of voltage samples in the pulse trace in $[\mu \mathrm{V}]$,

$\Delta T$ is the time resolution (sampling time) in [s],

$T$ is the total duration of the pulse in [s],

$f$ is the frequency in $[\mathrm{Hz}]$,

$k_{A T T}$ is the attenuation of the cable + attenuator between the generator and oscilloscope.

Example of the IGUU2916 pulse shape in the C/D band is shown in Fig. 8 for changing the pulse repetition rate.
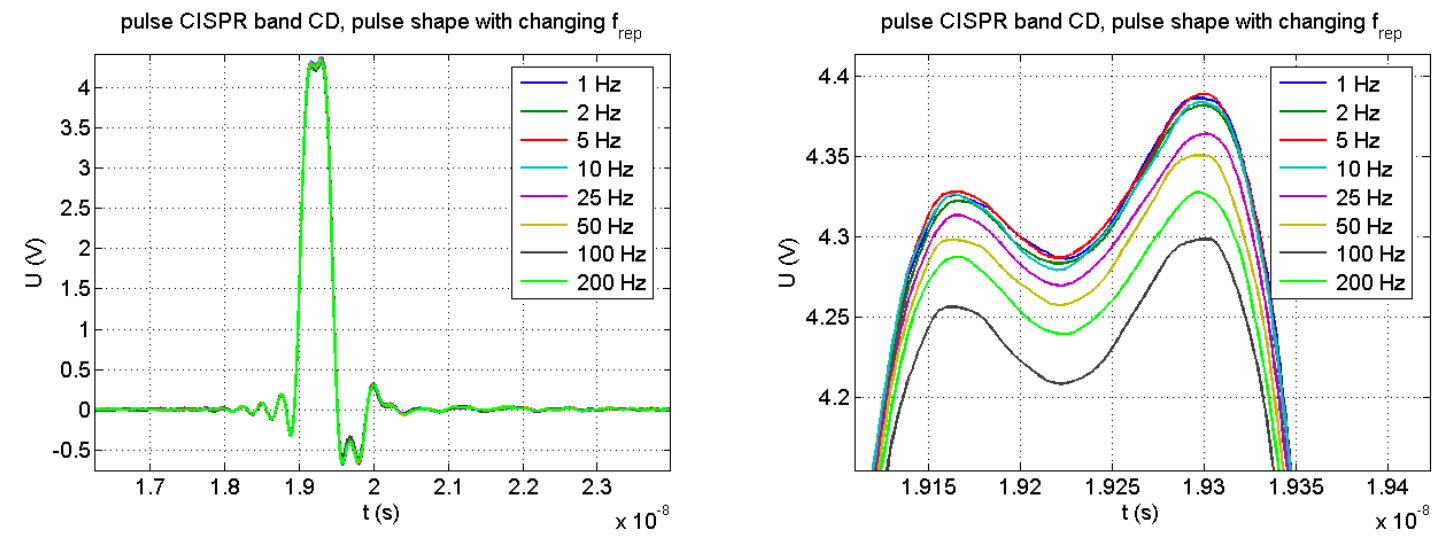

Fig. 8 Pulse shape for the band C/D of the IGUU2916 Main generator with changing pulse repetition rate, detail of the pulse maximum on the right (amplitude without correction for the cable and attenuators).

It can be seen from Fig. 8 the pulse shape in band C/D is distorted and moreover, it contains more noise than in case of the pulses in bands A and B (not shown). The results are shown in Tab. 4. It can be seen that do not correspond very well with the results of the Fourier transform method or the IF pulse measurement method. The measurement uncertainty mainly comprises of the cable + attenuator correction, impedance mismatch and type A uncertainty. It is obvious that this method is better suitable for pulse-modulated RF generators and not the base-band pulse generators like IGUU 2916.

The reason for the large deviation of some results from the results of other methods may be the presence of noise and undershoots or overshoots in the pulse trace. It can be shown that the contribution of these components to the total surface in $[\mathrm{V} \cdot \mathrm{s}]$ is significant. The signal is noisy and especially in band $\mathrm{C} / \mathrm{D}$, the error can be reduced by summing over only those samples, which correspond to the pulse (the choice of samples may be subjective). 


\section{Measurement of one spectrum line amplitude}

The principle of this method is a comparison of one spectrum line of the pulse signal with a known CW signal spectrum (equal frequency). This method assumes the generator pulse repetition frequency is high enough so that only one spectral line falls within the EMI receiver filter bandwidth, see Fig. 9. The nominal filter bandwidths for the $6 \mathrm{~dB}$ amplitude drop are $200 \mathrm{~Hz}$ (band A), $9 \mathrm{kHz}$ (band B) and $120 \mathrm{kHz}$ (band C/D), respectively. This method is referred to as "harmonic measurement" in [5].

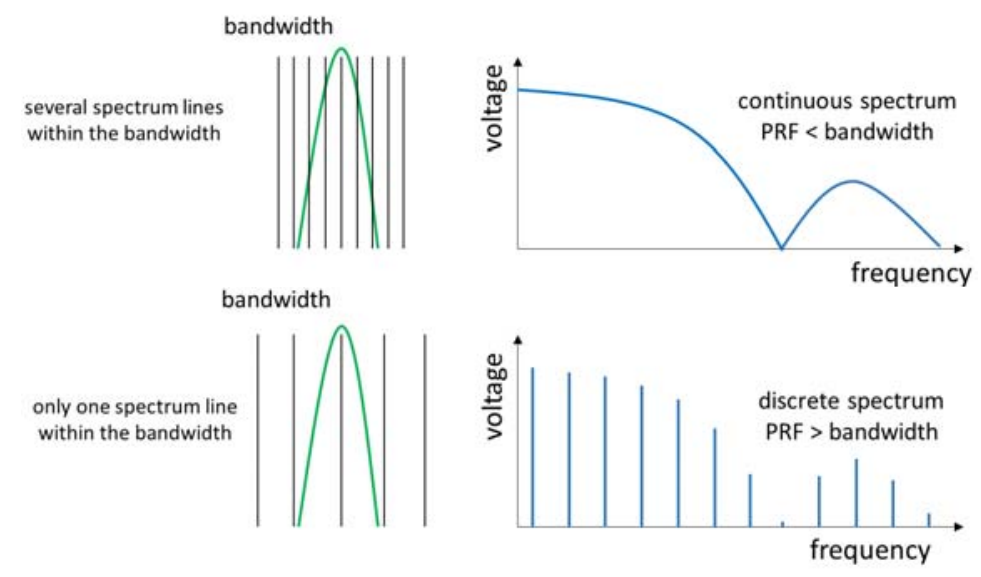

Fig. 9 Measurement of one spectrum line amplitude ( $\mathrm{PRF}=$ pulse repetition frequency).

The spectrum amplitude is then calculated using a simple formula

$$
S(f)=\frac{A}{f_{\text {rep }}},
$$

where $A$ is the substitution $\mathrm{CW}$ signal level for the same reading of the receiver and $f_{\text {rep }}$ is the generator pulse repetition frequency. A calibrated measuring receiver is needed in this case. The measurement setup is shown in Fig. 10. The receiver filter should be well symmetrical.

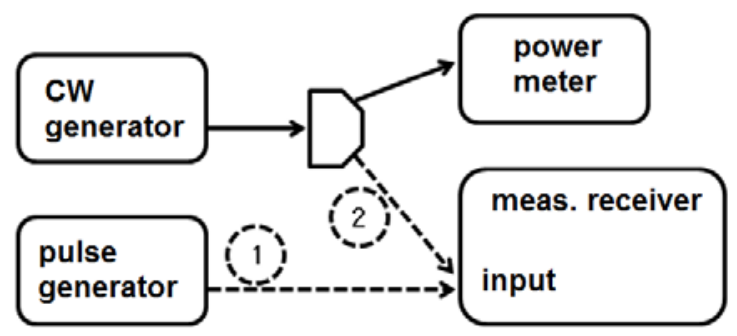

Fig. 10 Measurement setup for the measurement of one spectrum line amplitude. 
The maximum pulse repetition rate of the IGUU2916 main generator is $200 \mathrm{~Hz}$, thus only the band A could be possible to verify. In other bands, the pulse repetition frequency would have to be sufficient. Results of this method are not compared with the other methods in Tab. 4.

\section{Conclusion}

In this paper, various methods for calibration of pulse generators are discussed and results of measurement of the spectrum amplitude of a particular pulse generator Schwarzbeck IGUU 2916 are presented. The spectrum amplitude was evaluated using following methods: (a) Fourier transform of the time-domain pulse waveform; (b) intermediate-frequency measurement method; (c) measurement of pulse amplitude and duration and (d) measurement of one spectrum line amplitude. The method (a) can achieve the lowest measurement uncertainty and is simple to perform. It requires fast digital real-time or sampling oscilloscope and the spectrum of the measured waveform must be corrected for known attenuation of the signal path. The method (b) uses an EMI receiver which is more common in calibration laboratories. The measurement uncertainty is comparable to the method (a). One has to be careful and not to overload the receiver input, as time-domain pulse generators use high peak amplitudes which may destroy the input mixer. Method (c) is more suitable for pulsemodulated RF generators and the results for a base-band pulse generator IGUU 2916 band $\mathrm{C} / \mathrm{D}$ are not reliable due to the distorted pulse shape. The measurement uncertainty is slightly higher than that of methods (a), (b). Method (d) is applicable only for pulse generators with very high pulse repetition rates (at least $500 \mathrm{~Hz}$ for band A, at least $10 \mathrm{kHz}$ for band $\mathrm{B}$ and at least $120 \mathrm{kHz}$ for band C/D). The results achieved for band A and B of the IGUU 2916 Aux generator are comparable to method (a) for the same generator, however, the measurement uncertainty is rather high due to noise and low repeatability of the pulses. The goal uncertainty of maximum $\pm 0.5 \mathrm{~dB}$ given in standards could not be achieved using method (d). The measured results of methods (a) to (c) with measurement uncertainties are summarized in Tab. 4. 


\begin{tabular}{|c|c|c|c|c|c|c|c|}
\cline { 2 - 8 } \multicolumn{2}{c|}{} & \multicolumn{2}{c|}{ Method A } & \multicolumn{2}{c|}{ Method B } & \multicolumn{2}{c|}{ Method C } \\
\hline \multirow{4}{*}{ Band } & $\begin{array}{c}\text { Frequency } \\
(\mathrm{MHz})\end{array}$ & $S(f)$ & Unc. & $S(f)$ & Unc. & $S(f)$ & Unc. \\
\cline { 2 - 8 } & 0.009 & 139.90 & 0.13 & 139.84 & 0.21 & 139.81 & 0.22 \\
\cline { 2 - 8 } A & 0.01 & 139.83 & 0.13 & 139.87 & 0.21 & 139.81 & 0.22 \\
\cline { 2 - 8 } & 0.05 & 139.77 & 0.12 & 139.83 & 0.21 & 139.68 & 0.22 \\
\cline { 2 - 8 } & 0.1 & 139.79 & 0.11 & 139.84 & 0.21 & 139.24 & 0.22 \\
\cline { 2 - 8 } & 0.15 & 139.79 & 0.11 & 140.25 & 0.21 & 138.49 & 0.22 \\
\hline \multirow{4}{*}{ B } & 0.15 & 107.11 & 0.21 & 107.40 & 0.12 & 106.86 & 0.22 \\
\cline { 2 - 8 } & 0.6 & 106.90 & 0.23 & 107.20 & 0.12 & 106.86 & 0.22 \\
\cline { 2 - 8 } & 1 & 106.93 & 0.20 & 107.06 & 0.12 & 106.85 & 0.22 \\
\cline { 2 - 8 } & 10 & 106.94 & 0.22 & 107.05 & 0.12 & 105.95 & 0.22 \\
\hline & 30 & 106.75 & 0.22 & 106.54 & 0.12 & 96.40 & 0.23 \\
\hline & 50 & 90.08 & 0.16 & 89.62 & 0.14 & 90.94 & 0.25 \\
\cline { 2 - 8 } & 120 & 90.04 & 0.17 & 89.20 & 0.14 & 85.85 & 0.25 \\
\hline & 300 & 90.14 & 0.16 & 88.96 & 0.14 & 78.35 & 0.25 \\
\hline & 500 & 90.12 & 0.18 & 88.57 & 0.14 & 73.91 & 0.25 \\
\hline & 1000 & 89.57 & 0.20 & 88.61 & 0.15 & 45.79 & 0.26 \\
\hline
\end{tabular}

Tab. 4 Summary of measured results using different methods. IGUU 2916 Main generator, amplitude setting $60 \mathrm{~dB} \mu \mathrm{V}$. The spectrum amplitude $S(f)$ is given in $\mathrm{dB} \mu \mathrm{V} / \mathrm{MHz}$ and the associated measurement uncertainty is given in $\mathrm{dB}(\mathrm{k}=2)$.

Method A = Fourier transform of the time-domain pulse waveform 
Method B = Intermediate-frequency measurement method

Method $\mathrm{C}=$ Measurement of pulse amplitude and duration

\section{Acknowledgement}

This work was supported by the project 15RPT01 RFMicrowave. This project has received funding from the EMPIR programme co-financed by the Participating States and from the European Union's Horizon 2020 research and innovation programme.

\section{References}

[1] M. A. Azpúrua, M. Pous, J. A. Oliva, B. Pinter, M. Hudlička and F. Silva, "Waveform Approach for Assessing Conformity of CISPR 16-1-1 Measuring Receivers," IEEE Transactions on Instrumentation and Measurement, vol. 67, no. 5, pp. 1187-1198, May 2018.

[2] G. Mahesh, J. T. Vethamoni, M. Vasanthakumar and V. Venkatesan, "Pulse response analysis of quasi-peak detector in EMI receiver," 2015 13th International Conference on Electromagnetic Interference and Compatibility (INCEMIC), Visakhapatnam, 2015, pp. 14-18.

[3] M. A. Azpúrua, M. Pous and F. Silva, "Specifying the Waveforms for the Calibration of CISPR 16-1-1 Measuring Receivers," IEEE Transactions on Electromagnetic Compatibility, 2019 (Early Access) . doi: 10.1109/TEMC.2019.2923813

[4] S. Pasakawee and V. Sittakul, "Calibration and uncertainty evaluation of pulse generator for EMC testing using FFT technique," 2016 13th International Conference on Electrical Engineering/Electronics, Computer, Telecommunications and Information Technology (ECTI-CON), Chiang Mai, 2016, pp. 1-5.

[5] Specification for radio disturbance and immunity measuring apparatus and methods Part 1-1: Radio disturbance and immunity measuring apparatus - Measuring apparatus. EN 55016-1-1 ed. 3 standard, 2014.

[6] CISPR 16-1-1 Ed. 4: Specification for radio disturbance and immunity measuring apparatus and methods - Part 1-1: Radio disturbance and immunity measuring apparatus Measuring apparatus, International Electrotechnical Commission, IEC, 2015.

[7] R. B. Andrews, "An Impulse Spectral Intensity Measurement System," IEEE Transactions on Instrumentation and Measurement, vol. 15, no. 4, pp. 299-303, Dec. 1966. 
[8] N. G. Paulter, and D. R. Larson, "Impulse spectrum amplitude uncertainty analysis," Metrologia, vol. 43, no. 6, pp. 477-485, 2006.

[9] Andrews, J. R.; Arthur, M. G.: Spectrum Amplitude - Definition, Generation and Measurement; National Bureau of Standards; 1977

[10] IEEE Standard for the Measurement of Impulse Strength and Impulse Bandwidth; IEEE Std. 376-1975 (inactive - withdrawn)

[11] Instruction Manual for VLF/HF/VHF/UHF Calibration Pulse and Sine Wave Generator IGUU 2916, Schwarzbeck Mess Elektronik, Rev. B [online]. Available: http://schwarzbeck.de/Datenblatt/m2916.pdf

[12] MIL-STD-462, Military Standard: Measurement of Electromagnetic Interference Characteristics, 1967.

[13] J. R. Palladino, "A New Method for the Spectral Density Calibration of Impulse Generators," IEEE Transactions on Electromagnetic Compatibility, vol. EMC-13, no. 1, pp. 2-7, Feb. 1971.

\section{Biography}

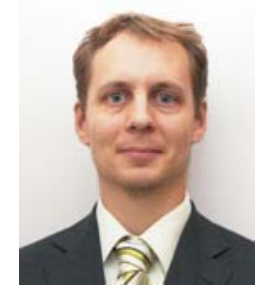

Martin Hudlička (S'04-M'08-SM'15) received the Ing. (M.Sc.) and Ph.D. degrees in electrical engineering from the Czech Technical University, Prague, Czech Republic, in 2004 and 2007, respectively. In 2007, he joined the Department of Primary Metrology of RF Electrical Quantities, Czech Metrology Institute, Prague, where he works as metrologist and researcher. His current research interests include microwave and millimeter-wave measurements and modern communication technologies.

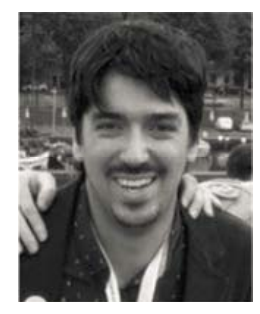


Marco A. Azpúrua (S'07-M'13-SM'-19) received the B.Sc. degree in telecommunications engineering, in 2008, and the M.Sc. degree in electrical engineering from the Universidad Central de Venezuela, Caracas, in 2013. In 2018, received the Ph.D. degree in Electronics Engineering from the Universitat Politècnica de Catalunya (UPC), Barcelona, Spain, for his contributions to Full Time Domain EMI measurements and their applications in complex scenarios. Currently, he is working as Researcher with the Electromagnetic Compatibility Group (GCEM) of the UPC. Formerly, he was a Researcher in the Applied Electromagnetics Laboratory, Instituto de Ingeniería (FII), Caracas, Venezuela. His research interests include electromagnetic compatibility, antenna and microwave measurement technologies, estimation of measurement uncertainty in complex systems and validation methods.

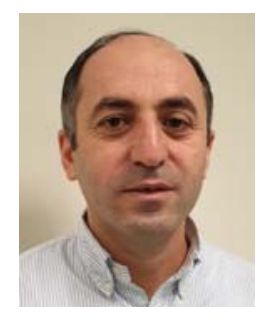

Murat Celep received the technician degree in electronics from the Tekirdag Vocational School, University of Trakya, Edirne, Turkey, in 1992, and the B.Sc., M.Sc., and Ph.D. degrees from the Department of Electronics and Communication Engineering, Kocaeli University, Izmit, Turkey, in 1999, 2004, and 2013, respectively. He joined the RF and Microwave Laboratory, TUBITAK Ulusal Metroloji Enstitüsü (UME), Gebze, Turkey, in 1997. He is involved in performing primary and secondary levels of power, S-parameters, impedance, attenuation, and noise measurements at RF and microwave frequencies, installing and characterizing measurement systems, and uncertainty calculations. 


$$
\text { Almon }
$$




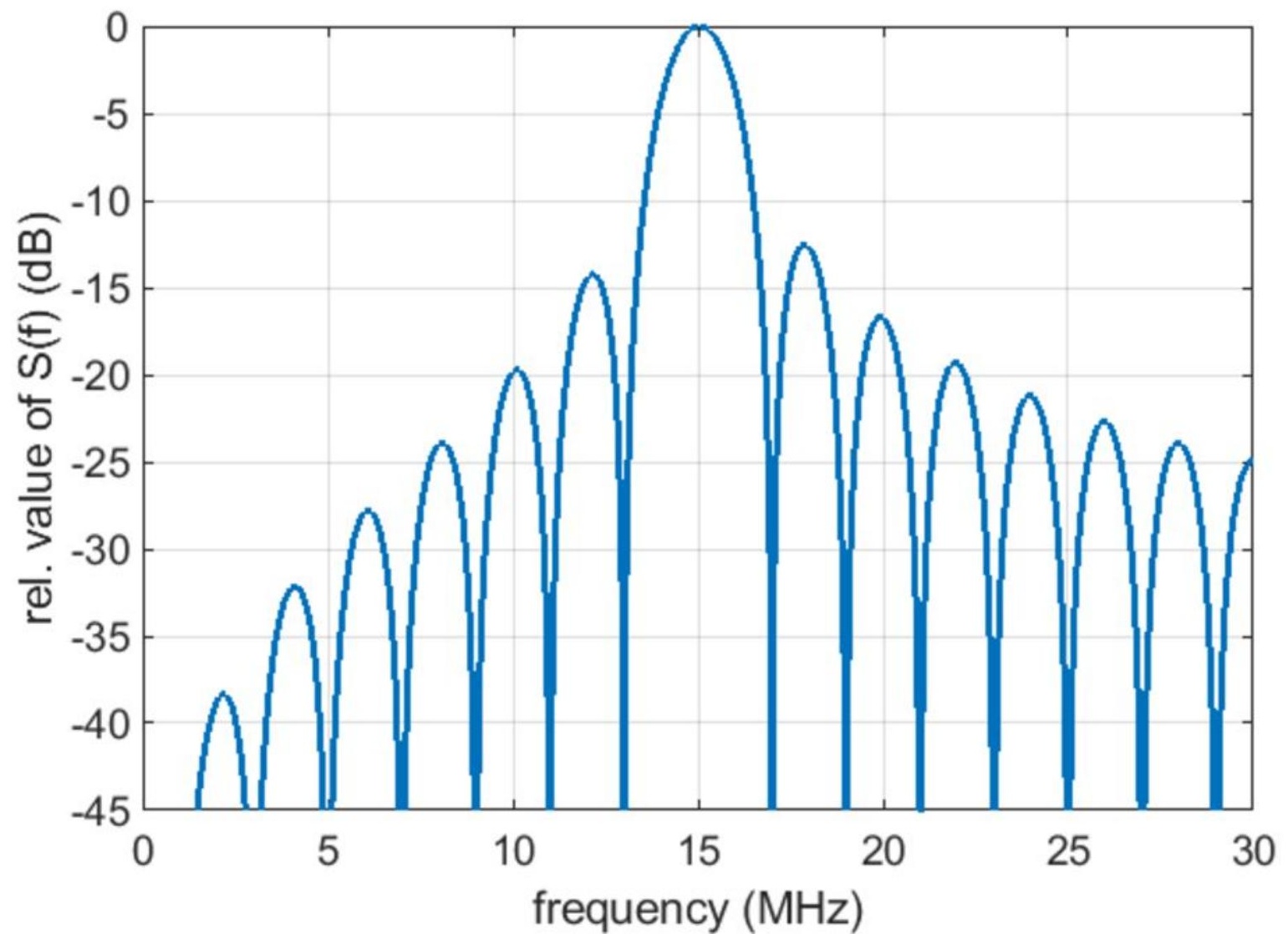




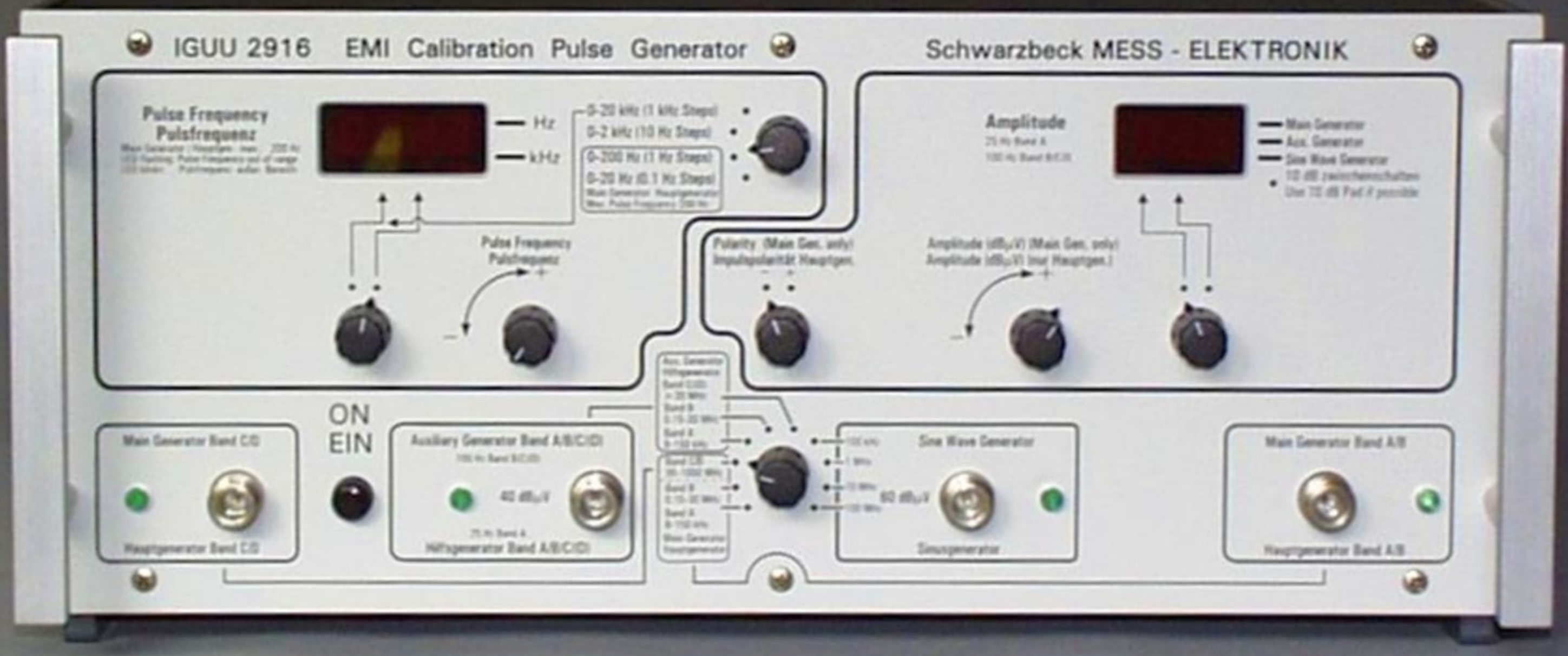


pulse generator

attenuator oscilloscope 


\section{pulse}

\section{oscilloscope} generator

trig in signal in

$$
\text { attenuator divider delay }
$$

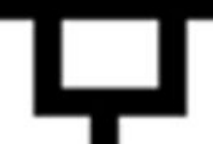




$$
\text { Almon }
$$



IF pulse CISPR band CD

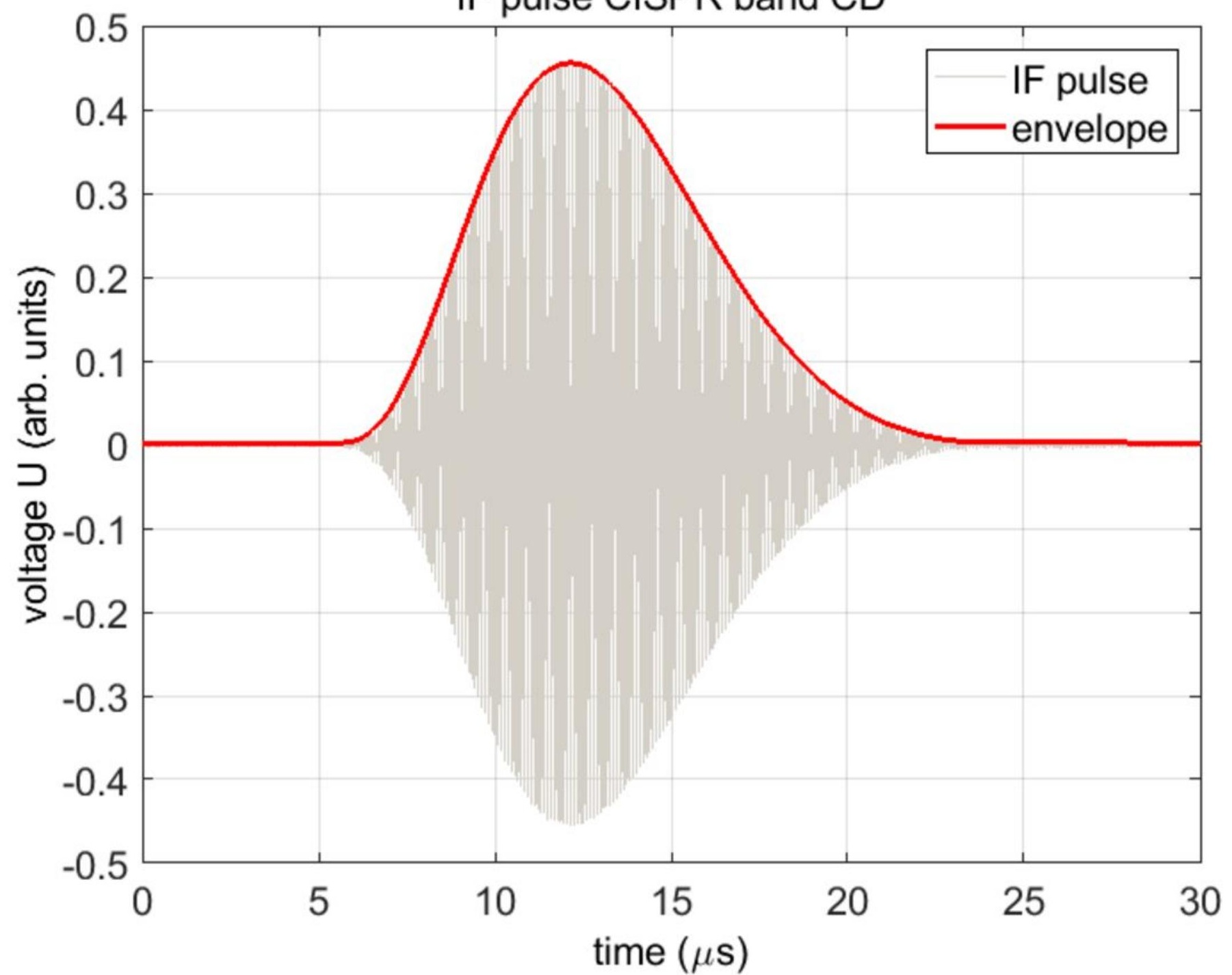


IF pulse CISPR band CD

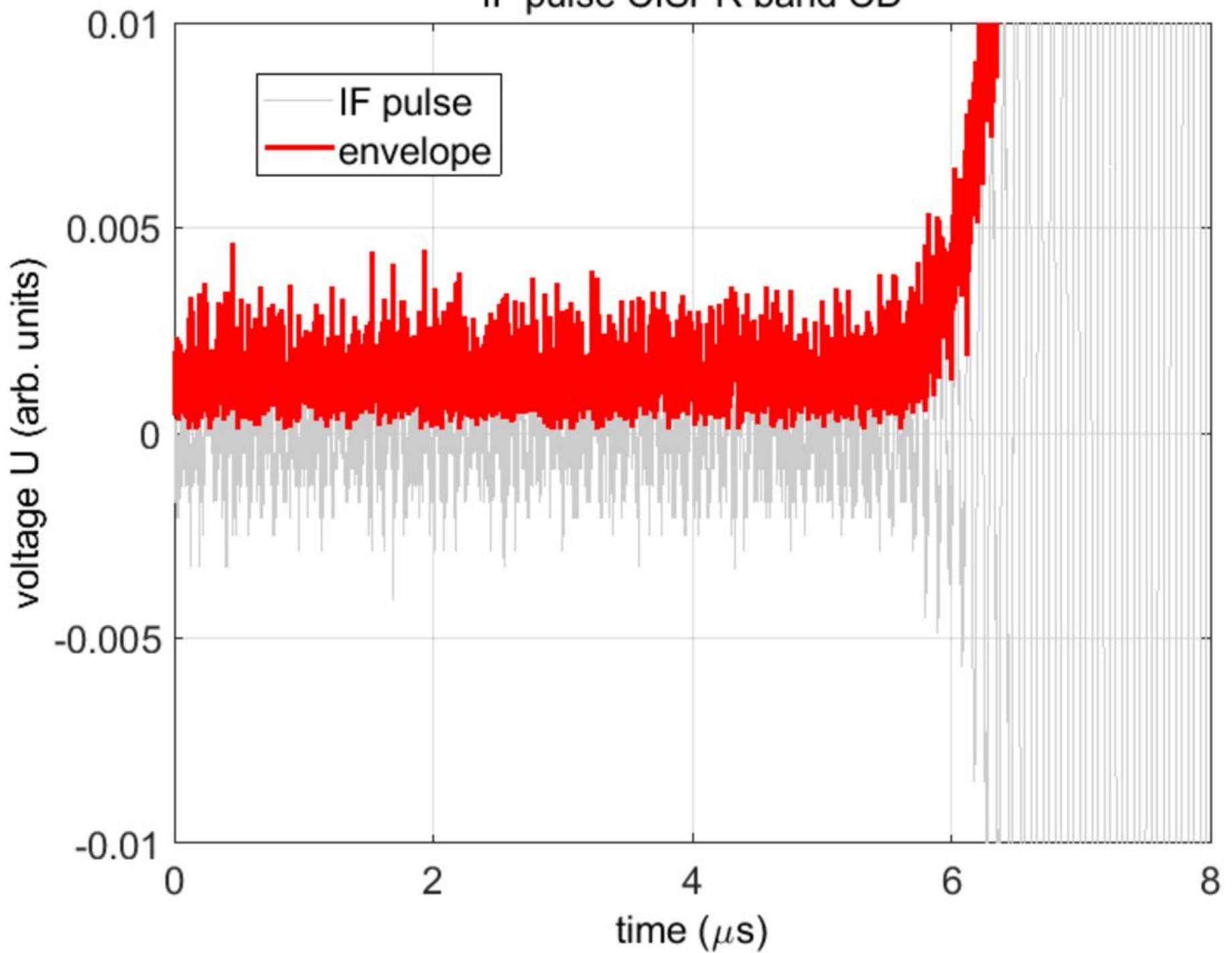




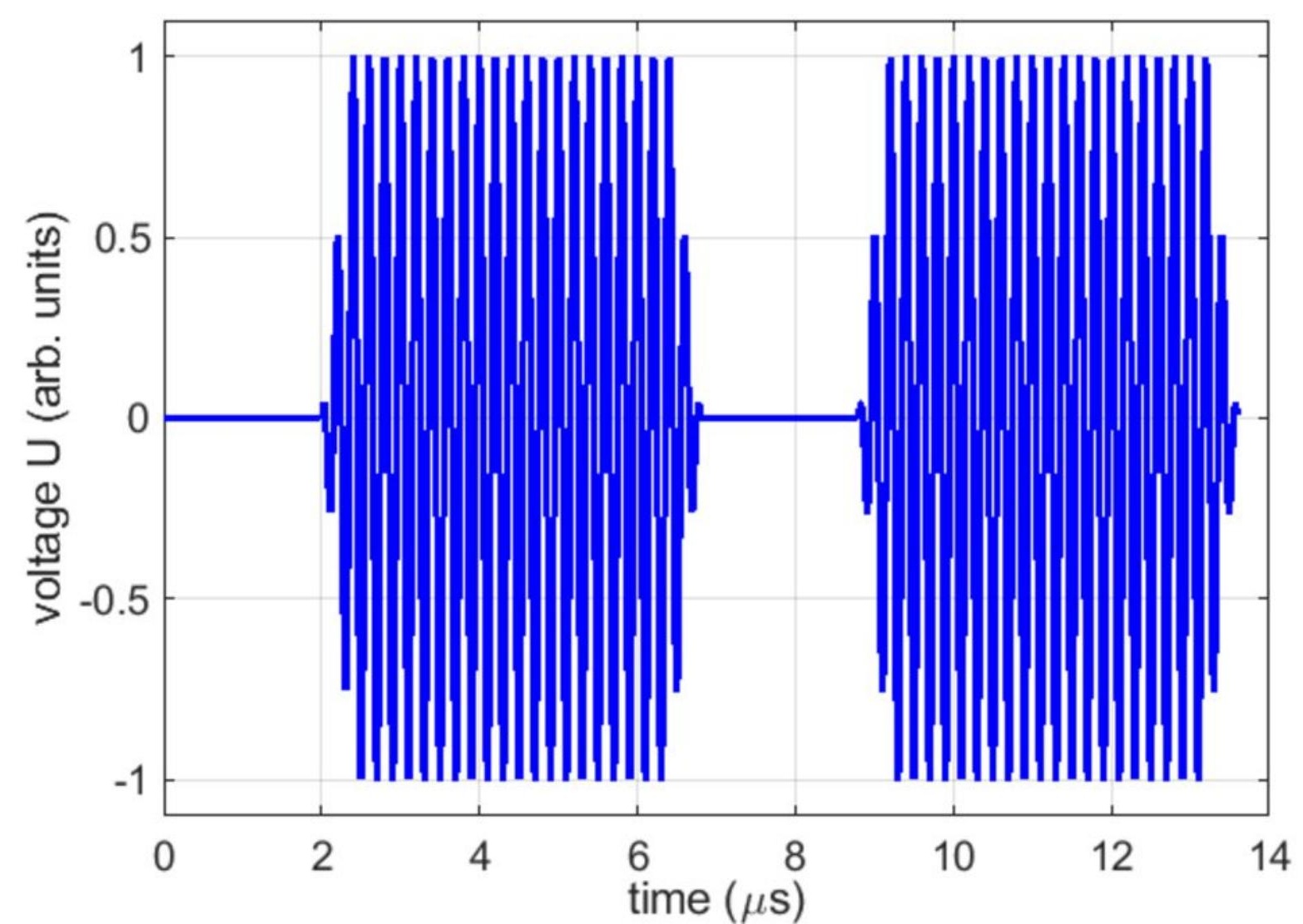


pulse CISPR band CD, pulse shape with changing $f_{\text {rep }}$

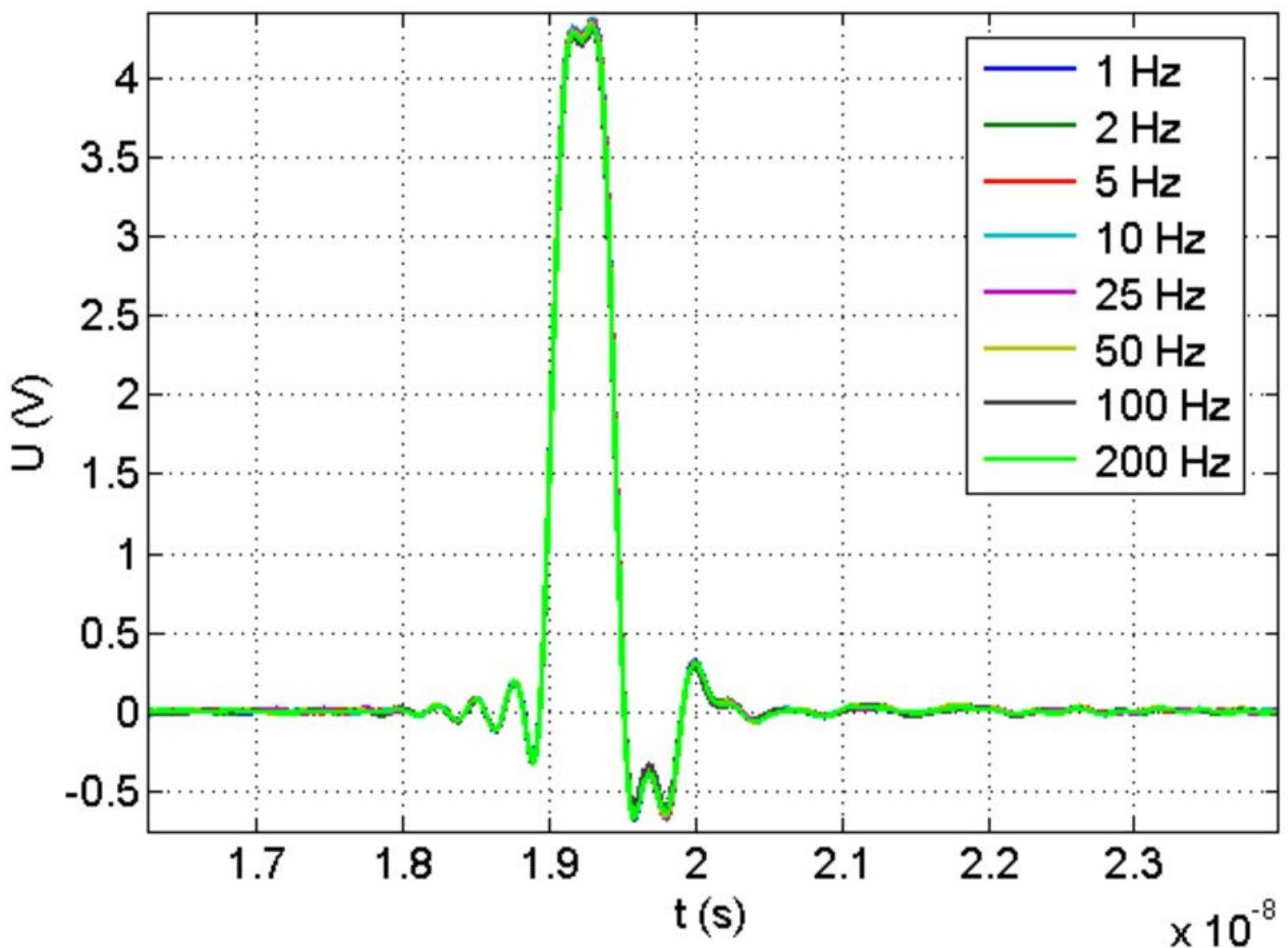


pulse CISPR band CD, pulse shape with changing $f_{r}$

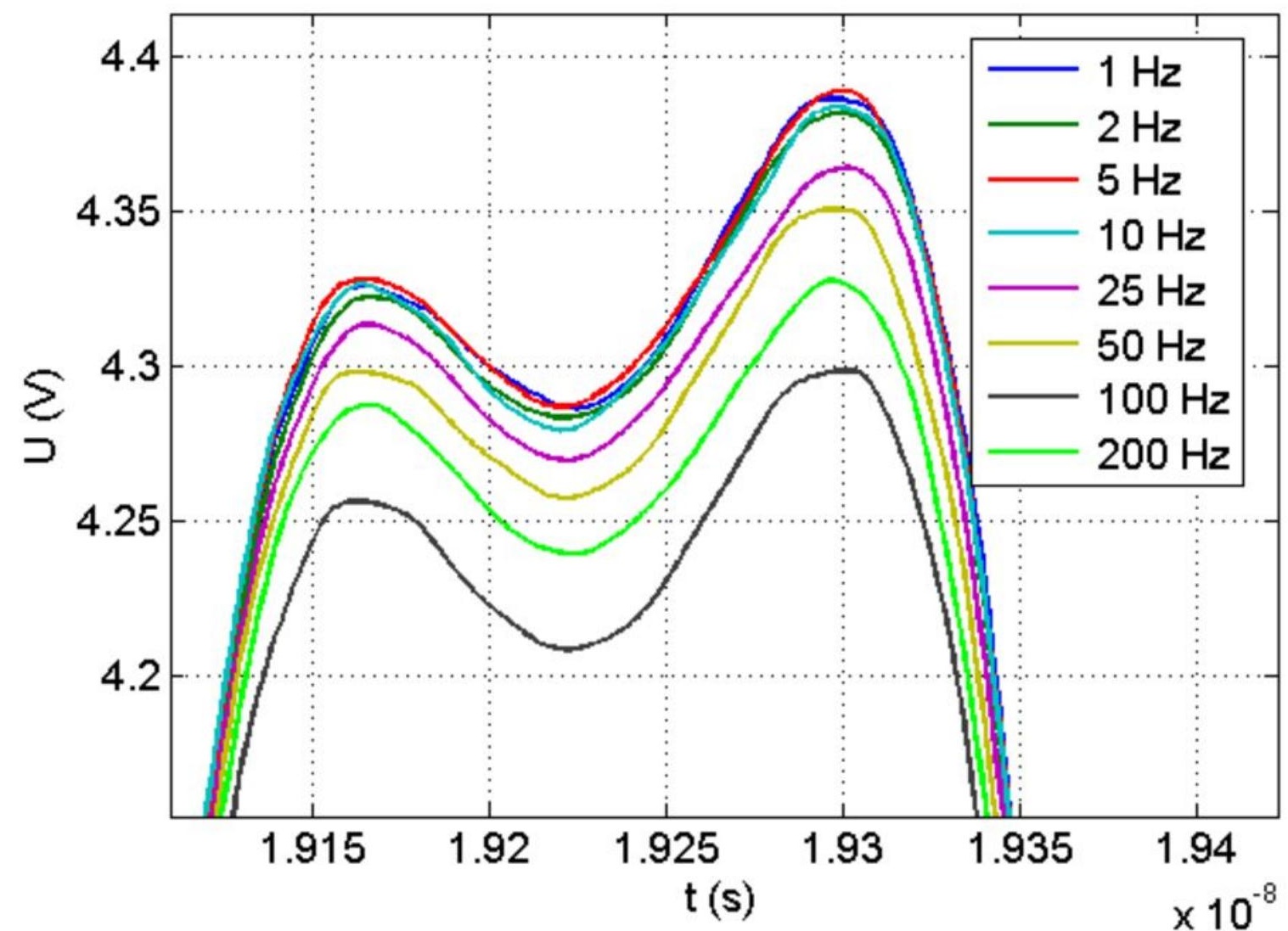




\section{bandwidth}

several spectrum lines within the bandwidth

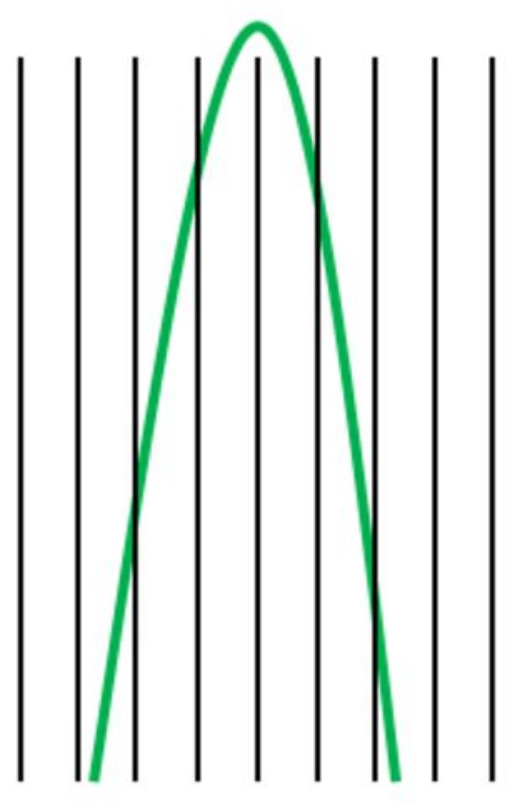

bandwidth

only one spectrum line within the bandwidth
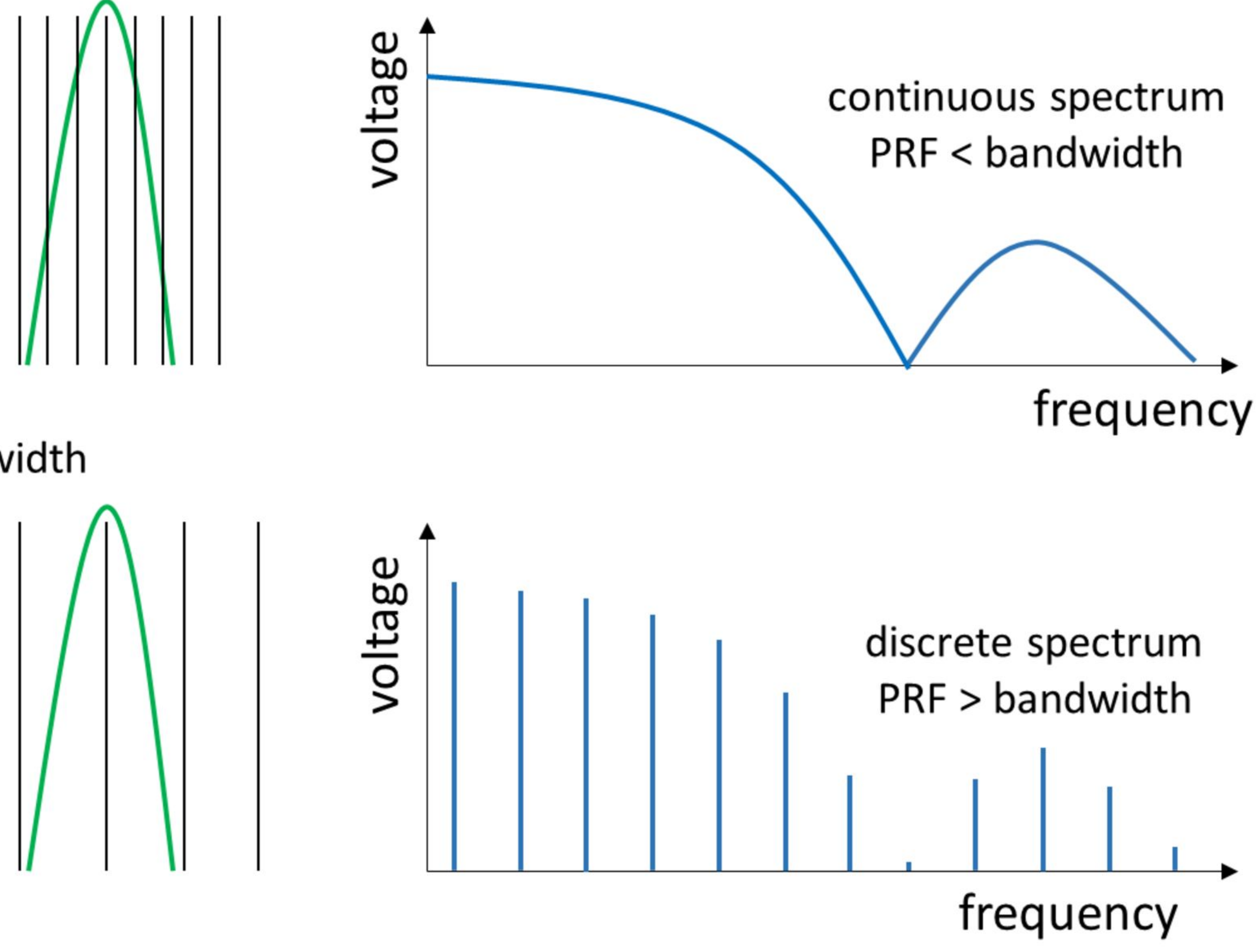


\section{CW generator}

power meter

pulse generator meas. receiver input 


\title{
Practical aspects of a pulse generator calibration
}

\author{
Martin Hudlička ${ }^{1}$, mhudlicka@cmi.cz, Marco A. Azpúrua ${ }^{2}$, Murat Celep $^{3}$ \\ ${ }^{1}$ Czech metrology institute, Brno, Czech Republic \\ ${ }^{2}$ Universitat Politècnica de Catalunya, Barcelona, Spain \\ ${ }^{3}$ TUBITAK Ulusal Metroloji Enstitüsü (UME), Gebze, Turkey
}

Measuring receivers are used for measurement of radio disturbance in the frequency range typically $9 \mathrm{kHz}$ to $18 \mathrm{GHz}$. Such receivers can be either electromagnetic interference (EMI) receivers or spectrum analyzers with the quasi-peak (QP) detector [1], [2]. Requirements for measuring receivers are discussed in European CISPR documents (Comité International Spécial des Perturbations Rádioélectriques) and US standards ANSI 63.2 (QP parts derived from CISPR). Although novel techniques are being evaluated [3], the traditional way of checking compliance of the receiver with requirements of these standards is using a calibrated pulse generator [4]. The calibration of pulse generators is discussed in the standard EN 550161-1 [5], which is the harmonized version of the international standard IEC/CISPR 16-1-1 (currently Ed. 4 [6]). In the standard [5], however, only a very brief description of the methods is given and technical details are hidden. The measurement uncertainty of the pulse generator characterization is not discussed in the standards, however it was discussed in several previous works [7], [8]. This paper aims to provide a more thorough description of particular calibration methods together with practical hints which may be useful for students, calibration engineers and practitioners.

\section{Pulse generators}

According to [5], a pulse generator is an instrument capable of generating time-domain rectangular pulses, or a pulse-modulated RF signal. Rectangular pulses are typically used for lower frequencies (bands A/B), pulse-modulated RF signals for higher frequencies (bands $\mathrm{C} / \mathrm{D})$ because of the risk of receiver damage due to high peak voltages. The base-band pulse generators usually comprise of an energy-storage device (electrostatic, magnetic field) and a switch which discharges a fraction or all of the energy into a load. The pulse-modulated RF generator uses a harmonic signal with a pulse envelope. The spectrum is similar to a rectangular pulse (upconverted to the carrier frequency $f_{c}$ ), maximum of the spectrum is at $f_{c}$. The spectrum is uniform in a given bandwidth, which implies that pulses with longer duration can be used with lower amplitudes compared to base-band pulse generators (lower risk of 
measuring receiver damage). The typical spectrum of a rectangular base-band pulse and a pulse-modulated RF signal is shown in Fig. 1.
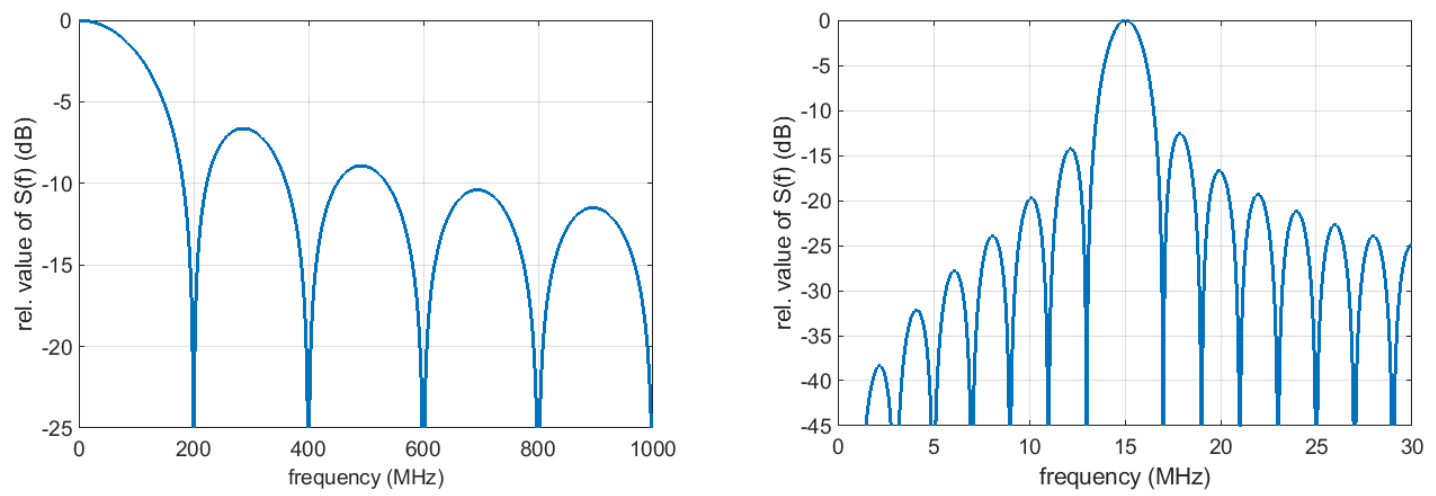

Fig. 1 Typical spectrum of a rectangular base-band pulse (left) and pulse-modulated RF signal (right).

The terminology used with the pulse generator characterization is summarized in Tab. 1 . All quantities for characterization of pulse generators have dimensional units $[\mathrm{V} / \mathrm{Hz}]$ or its mathematical equivalent.

\begin{tabular}{|c|c|c|}
\hline Quantity & Definition & Notes \\
\hline $\begin{array}{l}\text { Spectrum } \\
\text { amplitude }\end{array}$ & $S(f)=2|V(f)|$ & $\begin{array}{l}V(f) \text { is the Fourier transform of the } v(t) \text { signal in } \\
\text { the time-domain }[9],[10] \text {. It is the measure of the } \\
\text { amplitude spectrum at a certain frequency, } \\
\text { expressed in }[\mathrm{dB} \mu \mathrm{V} / \mathrm{MHz}] \text {. For a rectangular } \\
\text { pulse with amplitude } A \text { and duration } T \text { and low } \\
\text { frequencies }(f<<T) \text { we can write } S(f)=2 A T \text {. }\end{array}$ \\
\hline Impulse area & $A_{\text {imp }}=\int_{0}^{T} v(t) d t$ & $\begin{array}{l}\text { Voltage-time area of a pulse defined by the } \\
\text { integral. Sometimes referred to as impulse } \\
\text { strength, typically expressed in }[\mu \mathrm{Vs}] \text { or } \\
{[\mathrm{dB} \mu \mathrm{Vs}] \text {. Related to spectral density. } T \text { is one }} \\
\text { pulse period. }\end{array}$ \\
\hline Spectral intensity & $S(f)=2 A_{\text {imp }}$ & This definition can be found in [5] and [10]. \\
\hline Spectral density & $D=\sqrt{2} \times 10^{6} A_{i m p}$ & $\begin{array}{l}\text { Defined in }[5] \text {. Alternative term "impulse } \\
\text { strength" can be found. The term } \sqrt{ } 2 \text { stands for } \\
\text { the peak to RMS conversion. } D \text { is calculated in } \\
{[\mu \mathrm{V} / \mathrm{MHz}] \text { for } A_{\text {imp }} \text { given in }[\mu \mathrm{Vs}] \text {. }}\end{array}$ \\
\hline
\end{tabular}

Tab. 1 Quantities used for characterization of pulse generators. 


\section{Calibration methods}

The various pulse generator calibration methods will be demonstrated on the example calibration of the CISPR pulse generator IGUU2916 (base-band pulse generator, manufacturer Schwarzbeck, Germany). The generator is shown in Fig. 2. The methods are finally compared with regards to the measurement uncertainty, feasibility and required instrumentation. The polarity was always $(+)$ and the amplitude of the main generator was always $60 \mathrm{~dB} \mu \mathrm{V}$, the pulse repetition rate was changed according to the band.

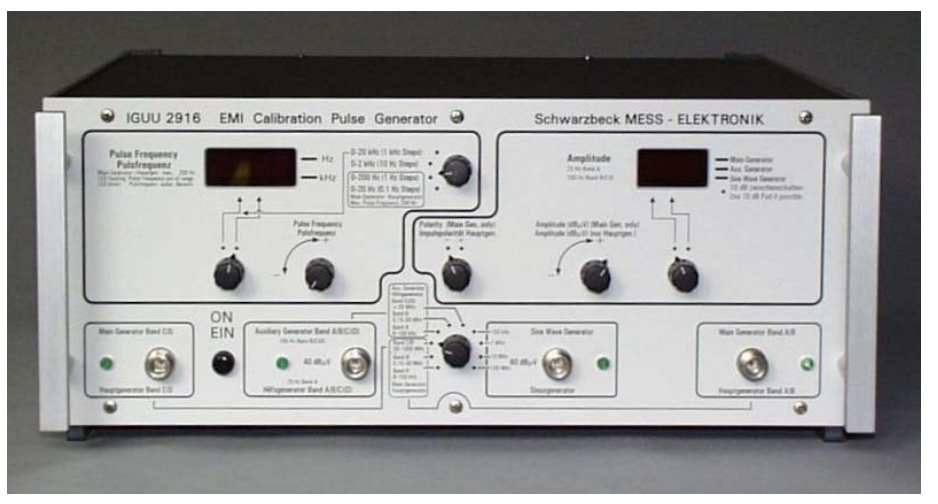

Fig. 2 CISPR pulse generator Schwarzbeck IGUU 2916.

In the EN 55016-1-1 [5] (CISPR 16-1-1 [6]) there are specified impulse areas of a typical generator for both open-circuit and $50 \Omega$ load, see Tab. 2. The values shown in this paper correspond to the measurement of the spectrum amplitude (calculated from the impulse area) into $50 \Omega$ nominal load.

\begin{tabular}{|l|c|c|c|c|}
\hline \multicolumn{1}{|c|}{ Band } & A & B & C & D \\
\hline $\begin{array}{l}\text { Frequency } \\
\text { range }\end{array}$ & $\{9-150\} \mathrm{kHz}$ & $\{0.15-30\} \mathrm{MHz}$ & $\{30-300\} \mathrm{MHz}$ & $\{300-1000\} \mathrm{MHz}$ \\
\hline $\begin{array}{l}\text { Impulse area } \\
\text { (open-circuit) }\end{array}$ & $13.5 \mu \mathrm{Vs}$ & $0.316 \mu \mathrm{Vs}$ & $0.044 \mu \mathrm{Vs}$ & $0.044 \mu \mathrm{Vs}$ \\
\hline $\begin{array}{l}\text { Impulse area } \\
\text { (into } 50 \Omega \\
\text { load) }\end{array}$ & $6.75 \mu \mathrm{Vs}$ & $0.158 \mu \mathrm{Vs}$ & $0.022 \mu \mathrm{Vs}$ & $0.022 \mu \mathrm{Vs}$ \\
\hline Repetition rate & $25 \mathrm{~Hz}$ & $100 \mathrm{~Hz}$ & $100 \mathrm{~Hz}$ & $100 \mathrm{~Hz}$ \\
\hline
\end{tabular}

Tab. 2 Impulse area of a generator for different bands specified in the EN 55016-1-1 standard. 


\section{Fourier transform of a time-domain pulse waveform}

The spectrum amplitude is determined by direct acquisition of the pulse generator output voltage using an oscilloscope and conversion into the frequency domain. For this purpose, a digital real-time oscilloscope (DRTO) or an equivalent-time sampling oscilloscope (DSO) can be used. The method is useful for base-band pulse generators, it is simple and time-efficient. Corrections for the cable (attenuator) properties and oscilloscope transfer function must be performed. A DRTO triggers directly the measured pulse. The traceability of DRTO is complicated due to the nonlinear behaviour of modern analogue-to-digital converters and the transfer function correction. A DSO needs an external trigger signal, which is usually derived from the measured signal itself (approx. $20 \mathrm{~ns}$ delay line is used). The measurement is traceable to the electro-optic sampling system. A general diagram of the measurement setup using the DRTO and DSO is shown in Fig. 3.
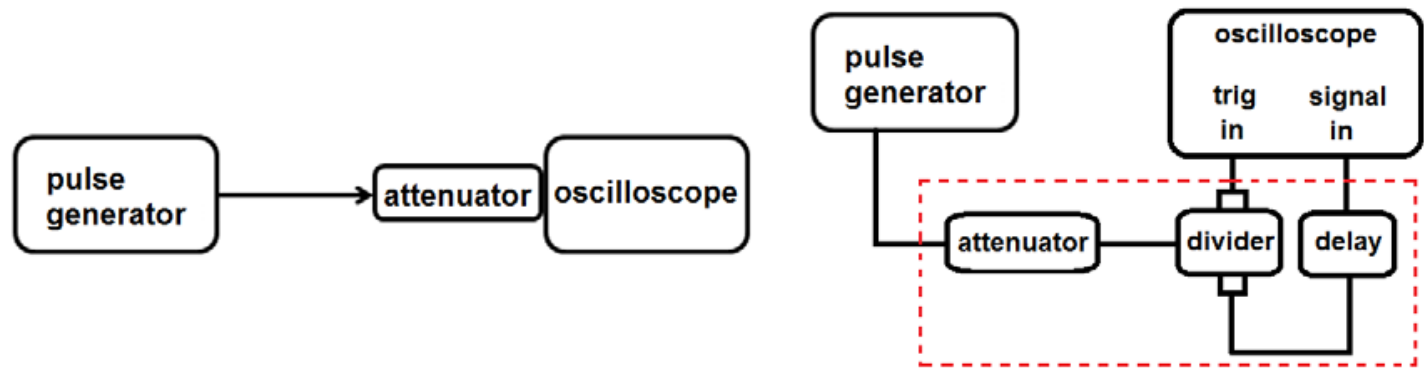

Fig. 3 Typical measurement setup with the use of a DRTO (left) and DSO (right).

The measurement equation is the following:

$$
S(f)=20 \log \left(\frac{|V(f)| \sqrt{2} \cdot 10^{6} \cdot k_{\text {ATT }} \cdot k_{\text {osc }}}{N_{F F T} \Delta f / 10^{6}}\right) \quad[\mathrm{dB} \mu \mathrm{V} / \mathrm{MHz}]
$$

where $V(f)$ is the Fourier transform of the voltage trace from oscilloscope in [V], $N_{F F T}$ is the FFT length, $\Delta f$ is the frequency resolution in $[\mathrm{Hz}], k_{A T T}$ is the total attenuation of the signal path, that is, the cables and external attenuators connected between the generator and oscilloscope, $k_{\text {osc }}$ is a factor taking into account the oscilloscope frequency response. A cable with attenuators on both sides should be used in order to reduce the pulse amplitude and improve the mismatch uncertainty.

Following text shows an example of measured and calculated results. The spectrum amplitude was calculated using (2) from oscilloscope voltage samples corrected for the cable and attenuator and oscilloscope transfer function. Following uncertainty contributions apply: 
- impedance mismatch correction between device 1 and 2 (e.g. between a generator and cable) $u_{M}=20 \log \left(1+\left|\Gamma_{1}\right|\left|\Gamma_{2}\right|\right)(\mathrm{dB})$, where $\Gamma_{1}$ and $\Gamma_{2}$ are linear reflection coefficients of devices 1 and 2, respectively,

- type A uncertainty was calculated from repeated calculations of the spectrum amplitude for all captured time traces, its value was determined from $n$ measurements in a standard way as

$$
u_{A y}=\sqrt{\frac{1}{n(n-1)} \sum_{i=1}^{n}\left(y_{i}-\bar{y}\right)} .
$$

The output reflection coefficient of the IGUU 2916 Main generator is not specified in the datasheet [11] and it was calculated from a hypothetical generator composed of an ideal voltage source with an inner resistance $R_{\text {in }}$ by measuring the output voltage (peak maximum) of the generator into two different load impedances $50 \Omega$ and $1000 \Omega$, respectively.

The measured results of the Fourier transform method with measurement uncertainty are summarized in Tab. 4. The example of measurement uncertainty evaluation for the Band A is given in Tab. 3.

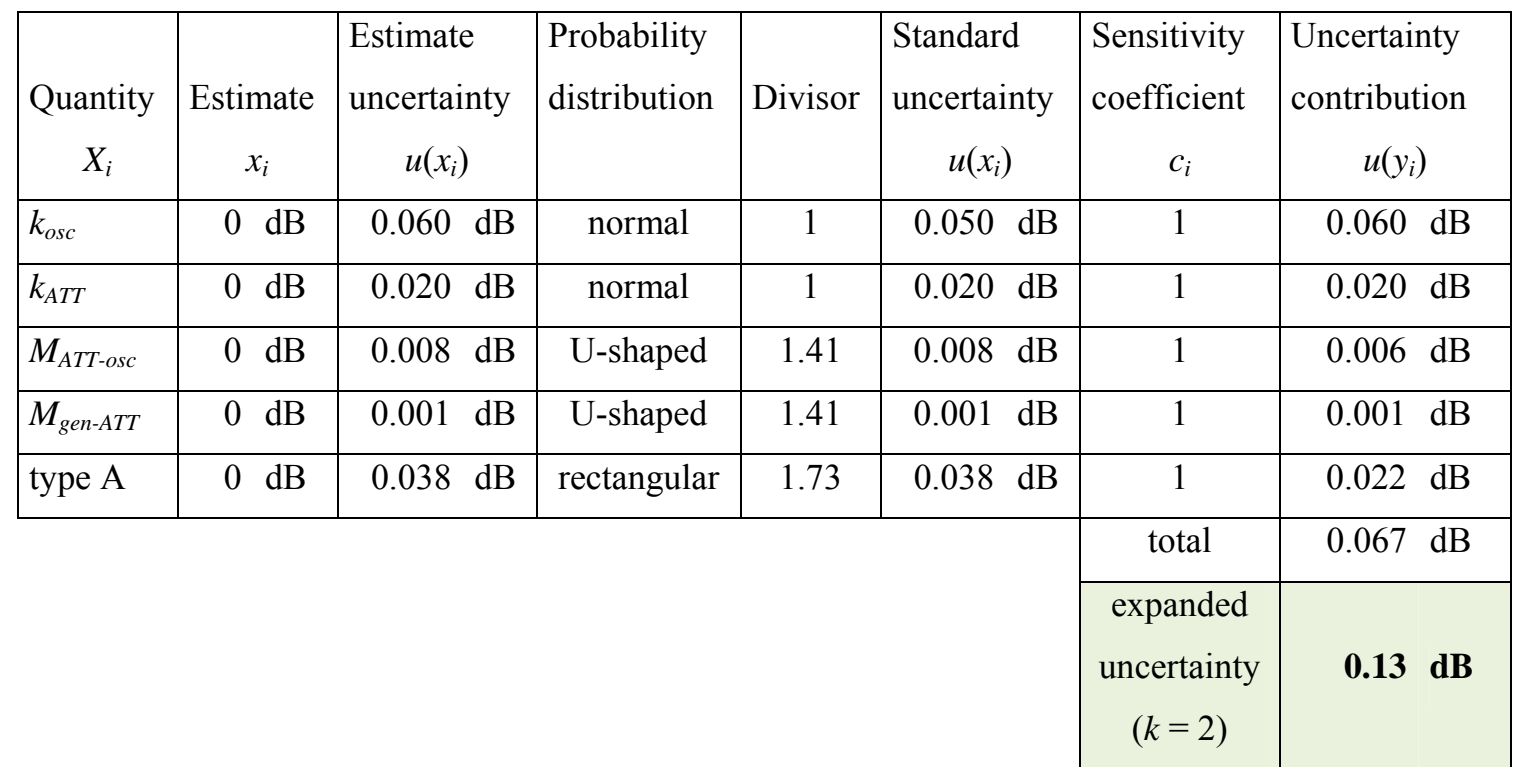

Tab. 3 Example of uncertainty calculation, band A, $f_{\text {rep }}=25 \mathrm{~Hz}$, frequency $9 \mathrm{kHz}$.

\section{Intermediate-frequency measurement method}

This method utilizes an EMI measuring receiver and its intermediate frequency output. It is referred to as "video pulse technique" in MIL-STD-462 [12], and it is referred to as "video pulse technique" and "area method" in [5]. The method uses a pulse signal and a reference CW signal (with known level) connected to a narrow-band filter, whereas the output of the 
filter (intermediate frequency) is acquired using an oscilloscope. The spectrum amplitude is then calculated from the response to both input signals at the frequency of the tuned filter (receiver) as follows

$$
S(f)=U_{r m s} / \mathrm{IBW}
$$

where $U_{r m s}(\mathrm{~V})$ is the level of $\mathrm{CW}$ signal which causes equal oscilloscope reading as the pulse signal, IBW $(\mathrm{Hz})$ is the impulse bandwidth of the used filter. The accuracy of the method is dependent on the accurate characterization of the receiver impulse bandwidth IBW [13]. The spectrum amplitude is calculated as the surface under the pulse envelope (i.e. positive amplitudes only), see Fig. 4. The measurement setup is shown in Fig. 5.

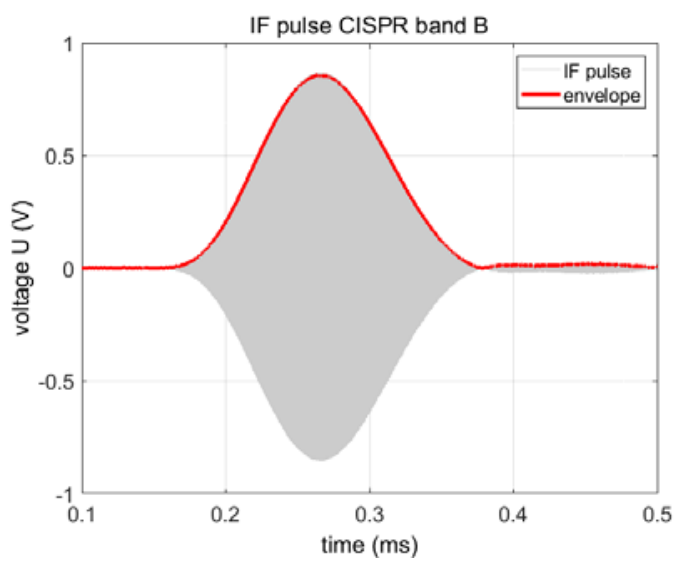

Fig. 4 Intermediate frequency measurement method.

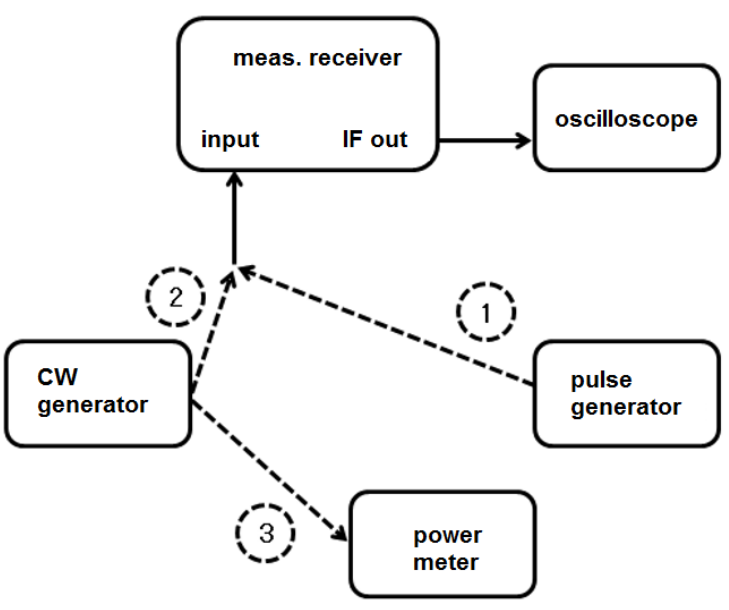

Fig. 5 Measurement setup of the intermediate frequency method. 
The practical procedure is following: in the first step, the pulse generator output is connected to the receiver input and the response of the receiver's IF filter is captured using an oscilloscope (direct connection using a high-grade cable, without attenuators). The peak-topeak amplitude of the trace is measured as well. In the second step, a CW sine signal is connected to the receiver and its amplitude is changed until the oscilloscope peak-to-peak reading is the same as for the pulse signal. The RMS level of this sine signal is measured using a calibrated power meter. The attenuation of the cable from the generator to the receiver and from the receiver IF output to the oscilloscope is not important, as it cancels due to the ratio measurement. The measurement equation is following

$$
S(f)=20 \log \left(\frac{2 V_{p w m, r m s} V_{e n v}}{V_{o s c, p p}} k_{p e a k} k_{o s c} k_{I B W} I B W \cdot 10^{6}\right) \quad[\mathrm{dB} \mu \mathrm{V} / \mathrm{MHz}]
$$

where $V_{p w m, r m s}$ is the voltage across a $50 \Omega$ load of the CW sine signal calculated from the RMS power measured by the power meter in $[\mu \mathrm{V}]$ $V_{e n v}$ is the amplitude of the IF pulse envelope (see the example in Fig. 6) in [V] $V_{\text {osc,pp }}$ is the peak-to-peak amplitude of the receiver response (IF output) to a CW sine signal measured by the oscilloscope in [V]

$I B W$ is the receiver impulse bandwidth in $[\mathrm{Hz}]$ $k_{\text {peak }}$ takes into account the uncertainty of the peak ratio of the response to pulse/CW signal (dimensionless)

$k_{\text {osc }}$ takes into account the oscilloscope frequency response (dimensionless) $k_{I B W}$ takes into account the uncertainty of the determination of the impulse bandwidth (dimensionless).

The receiver impulse bandwidth is calculated as [13]

$$
I B W=\frac{V_{e n v}}{X \cdot \frac{10}{N} \sum_{k=1}^{N} v_{e n v, k}}[\mathrm{~Hz}]
$$

where $V_{e n v}$ is the amplitude of the IF pulse envelope (see see the example in Fig. 6) in [V]

$X$ is the oscilloscope horizontal resolution in [s/div]

$N$ is the number of samples of the envelope [-]

$v_{e n v, k}$ represents the $k$-th sample of the IF pulse envelope in [V]

10 takes into account the number of oscilloscope horizontal screen divisions. 

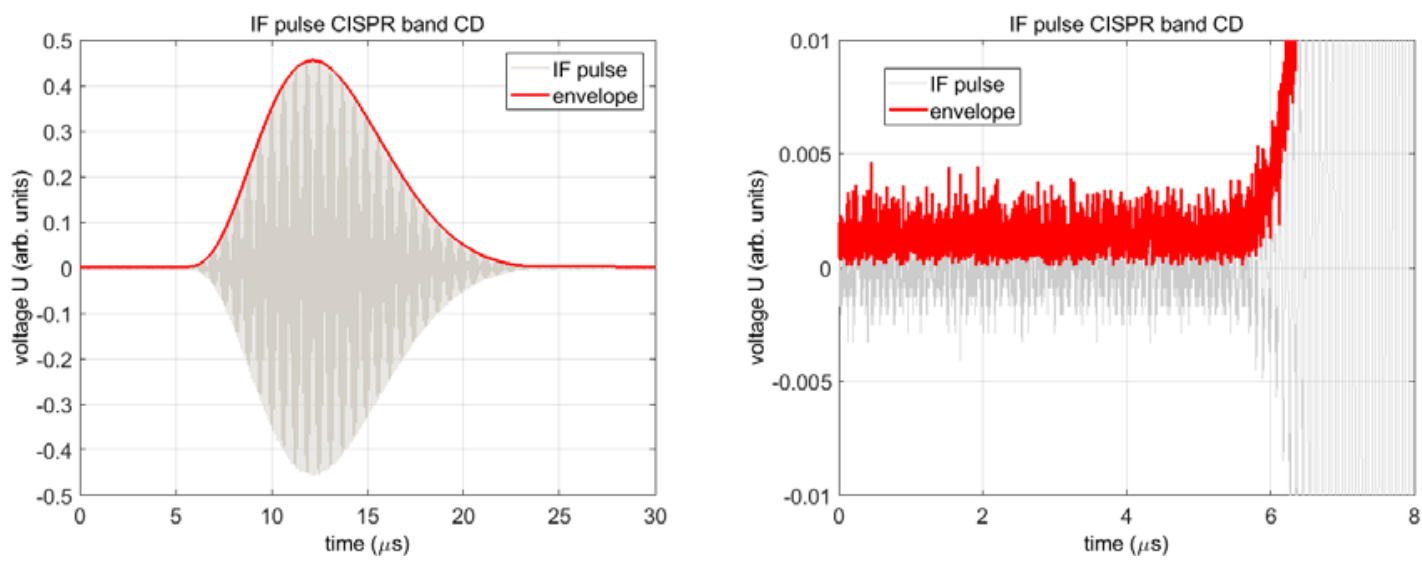

Fig. 6 Response of the receiver to a pulse signal + envelope of the signal (left), unfiltered envelope (right).

The determination of the IF pulse envelope can be done using different methods which results in slightly different calculated receiver impulse bandwidth and consequently spectrum amplitude. Either the envelope can be calculated as a moving average of the voltage IF trace (with e.g. 50 - 200 samples window), or it can be calculated as a magnitude of the Hilbert transform of the IF voltage trace (time-domain). It is convenient to filter the envelope trace using a low-pass filter in order to remove the noise (which is obvious in Fig. 6 right). The area under the envelope is then calculated as a sum of the voltage samples divided by the number of envelope samples. The spectrum amplitude was calculated using (6) from oscilloscope samples of the voltage at the IF output of an EMI receiver and their values together with the measurement uncertainty are given in Tab. 4.

\section{Measurement of pulse amplitude and duration}

This method uses an oscilloscope, whereas the pulse is sampled with high time-resolution. The method is most suitable for pulse-modulated RF generators. The spectrum amplitude is calculated from the area of the pulse $[\mathrm{V} \cdot \mathrm{s}]$. The ideal measurement equation can be written as follows

$$
S(f)=U_{r m s} \cdot T \cdot k_{c o r},
$$

where $U_{r m s}$ is the un-modulated CW signal level, $T$ is the modulation pulse duration and $k_{c o r}$ is a correction factor taking into account the signal level change after switching ON the pulse. The pulse repetition rate should be stable and correction factor should not change with $\mathrm{CW}$ frequency. An example of the pulse modulated $\mathrm{CW}$ signal with frequency $5 \mathrm{MHz}$ is shown in 
Fig. 7 (note the nonzero rising and trailing edge of the pulse due to the modulator nonidealities).

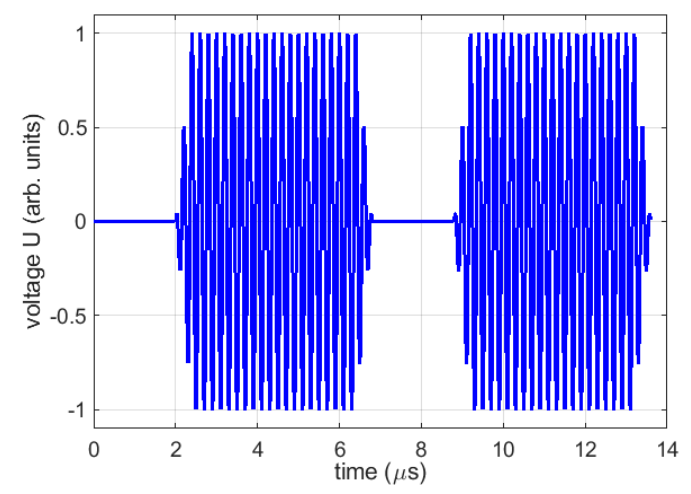

Fig. 7 Measurement of pulse amplitude and duration, pulse-modulated RF generator.

The method is also applicable for base-band pulse generators. The pulse shape must be very close to rectangular, which is not always true for the measured IGUU2916 generator (especially band C/D). The pulse amplitude and duration is measured using an oscilloscope. The pulse shape in the time domain is generally distorted by the transfer function of the cable + attenuator(s) and the oscilloscope transfer function. In order to remove this effect correctly, one has to perform deconvolution of the transfer function in the frequency domain. The pulse shape, however, was acquired using a short oscilloscope epoch (only the pulse visible on the screen), which makes the frequency resolution very coarse and the correction is not easily applicable.

$$
S(f)=20 \log \left(\sqrt{2} A \cdot k_{A T T} \cdot \Delta T \cdot\left|\frac{\sin (\pi f T)}{\pi f T}\right| \cdot 10^{6}\right) \quad[\mathrm{dB} \mu \mathrm{V} / \mathrm{MHz}],
$$

where $A$ is the sum of voltage samples in the pulse trace in $[\mu \mathrm{V}]$,

$\Delta T$ is the time resolution (sampling time) in [s],

$T$ is the total duration of the pulse in [s],

$f$ is the frequency in $[\mathrm{Hz}]$,

$k_{\text {ATT }}$ is the attenuation of the cable + attenuator between the generator and oscilloscope.

Example of the IGUU2916 pulse shape in the C/D band is shown in Fig. 8 for changing the pulse repetition rate. 

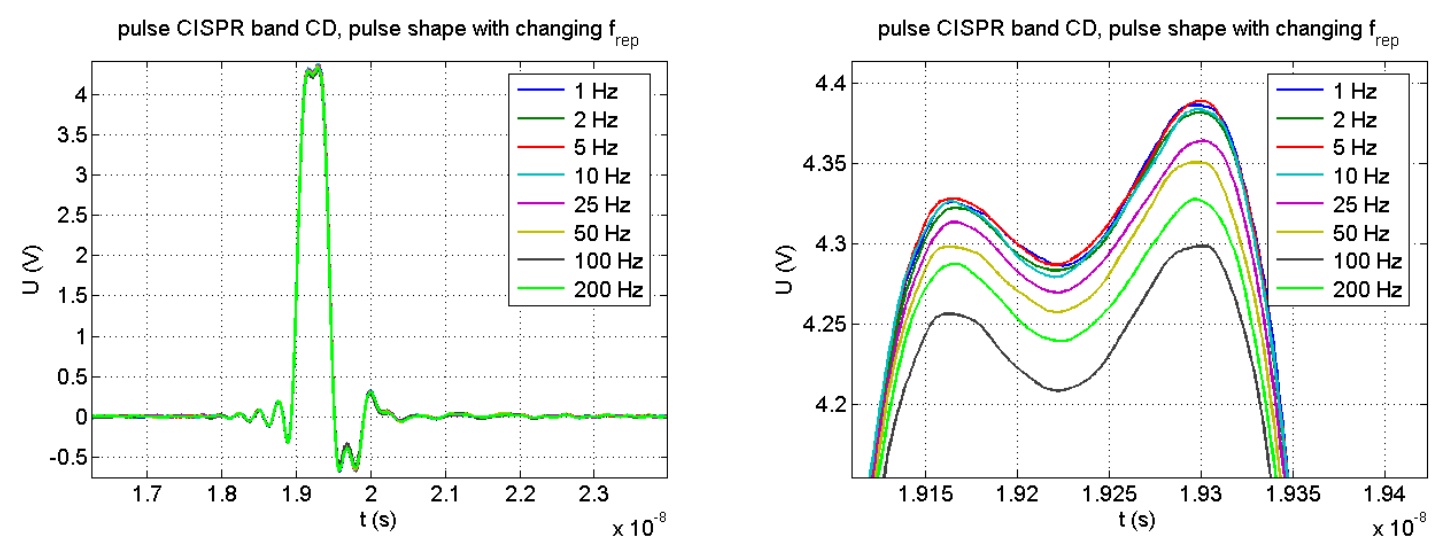

Fig. 8 Pulse shape for the band C/D of the IGUU2916 Main generator with changing pulse repetition rate, detail of the pulse maximum on the right (amplitude without correction for the cable and attenuators).

It can be seen from Fig. 8 the pulse shape in band C/D is distorted and moreover, it contains more noise than in case of the pulses in bands A and B (not shown).

The results are shown in Tab. 4. It can be seen that do not correspond very well with the results of the Fourier transform method or the IF pulse measurement method. The measurement uncertainty mainly comprises of the cable + attenuator correction, impedance mismatch and type A uncertainty. It is obvious that this method is better suitable for pulsemodulated RF generators and not the base-band pulse generators like IGUU 2916.

The reason for the large deviation of some results from the results of other methods may be the presence of noise and undershoots or overshoots in the pulse trace. It can be shown that the contribution of these components to the total surface in $[\mathrm{V} \cdot \mathrm{s}]$ is significant. The signal is noisy and especially in band $C / D$, the error can be reduced by summing over only those samples, which correspond to the pulse (the choice of samples may be subjective).

\section{Measurement of one spectrum line amplitude}

The principle of this method is a comparison of one spectrum line of the pulse signal with a known CW signal spectrum (equal frequency). This method assumes the generator pulse repetition frequency is high enough so that only one spectral line falls within the EMI receiver filter bandwidth, see Fig. 9. The nominal filter bandwidths for the $6 \mathrm{~dB}$ amplitude drop are $200 \mathrm{~Hz}$ (band A), $9 \mathrm{kHz}$ (band B) and $120 \mathrm{kHz}$ (band C/D), respectively. This method is referred to as "harmonic measurement" in [5]. 

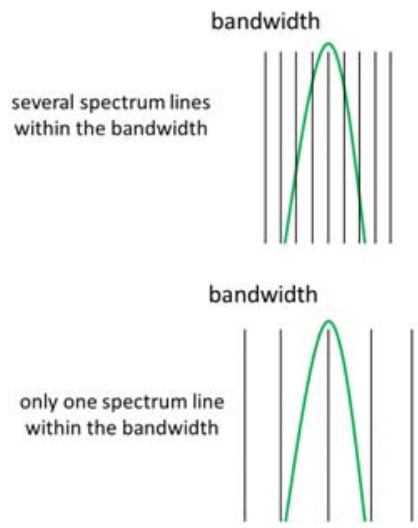
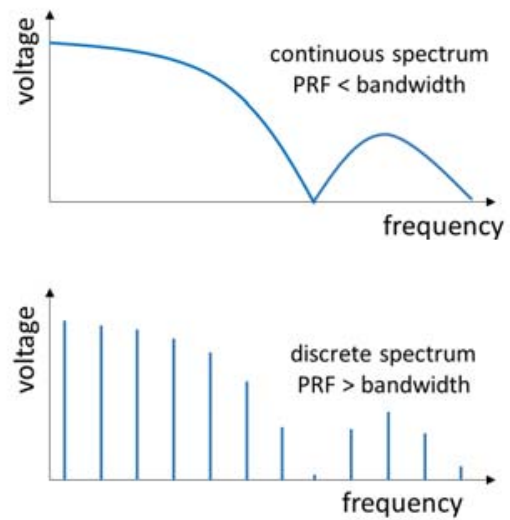

Fig. 9 Measurement of one spectrum line amplitude $(\mathrm{PRF}=$ pulse repetition frequency).

The spectrum amplitude is then calculated using a simple formula

$$
S(f)=\frac{A}{f_{\text {rep }}}
$$

where $A$ is the substitution $\mathrm{CW}$ signal level for the same reading of the receiver and $f_{\text {rep }}$ is the generator pulse repetition frequency. A calibrated measuring receiver is needed in this case. The measurement setup is shown in Fig. 10. The receiver filter should be well symmetrical.

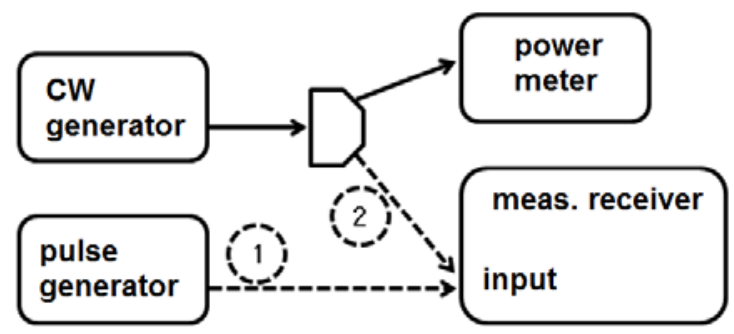

Fig. 10 Measurement setup for the measurement of one spectrum line amplitude.

The maximum pulse repetition rate of the IGUU2916 main generator is $200 \mathrm{~Hz}$, thus only the band A could be possible to verify. In other bands, the pulse repetition frequency would have to be sufficient. Results of this method are not compared with the other methods in Tab. 4.

\section{Conclusion}

In this guide, various methods for calibration of pulse generators are discussed and results of measurement of the spectrum amplitude of a particular pulse generator Schwarzbeck IGUU 2916 are presented. The spectrum amplitude was evaluated using following methods: (a) 
Fourier transform of the time-domain pulse waveform; (b) intermediate-frequency measurement method; (c) measurement of pulse amplitude and duration and (d) measurement of one spectrum line amplitude. The method (a) can achieve the lowest measurement uncertainty and is simple to perform. It requires fast digital real-time or sampling oscilloscope and the spectrum of the measured waveform must be corrected for known attenuation of the signal path. The method (b) uses an EMI receiver which is more common in calibration laboratories. The measurement uncertainty is comparable to method (a). One has to be careful and not to overload the receiver input, as time-domain pulse generators use high peak amplitudes which may destroy the input mixer. Method (c) is more suitable for pulsemodulated RF generators and the results for a base-band pulse generator IGUU 2916 band $\mathrm{C} / \mathrm{D}$ are not reliable due to the distorted pulse shape. The measurement uncertainty is slightly higher than that of methods (a), (b). Method (d) is applicable only for pulse generators with very high pulse repetition rates (at least $500 \mathrm{~Hz}$ for band A, at least $10 \mathrm{kHz}$ for band $\mathrm{B}$ and at least $120 \mathrm{kHz}$ for band C/D). The results achieved for band A and B of the IGUU 2916 Aux generator are comparable to method (a) for the same generator, however, the measurement uncertainty is rather high due to noise and low repeatability of the pulses. The goal uncertainty of maximum $\pm 0.5 \mathrm{~dB}$ given in standards could not be achieved using method (d). The measured results of methods (a) to (c) with measurement uncertainties are summarized in Tab. 4. 


\begin{tabular}{|c|c|c|c|c|c|c|c|}
\hline \multirow[b]{2}{*}{ Band } & \multirow[b]{2}{*}{$\begin{array}{c}\text { Frequency } \\
(\mathrm{MHz})\end{array}$} & \multicolumn{2}{|c|}{ Method A } & \multicolumn{2}{|c|}{ Method B } & \multicolumn{2}{|c|}{ Method C } \\
\hline & & $S(f)$ & Unc. & $S(f)$ & Unc. & $S(f)$ & Unc. \\
\hline \multirow{5}{*}{ A } & 0.009 & 139.90 & 0.13 & 139.84 & 0.21 & 139.81 & 0.22 \\
\hline & 0.01 & 139.83 & 0.13 & 139.87 & 0.21 & 139.81 & 0.22 \\
\hline & 0.05 & 139.77 & 0.12 & 139.83 & 0.21 & 139.68 & 0.22 \\
\hline & 0.1 & 139.79 & 0.11 & 139.84 & 0.21 & 139.24 & 0.22 \\
\hline & 0.15 & 139.79 & 0.11 & 140.25 & 0.21 & 138.49 & 0.22 \\
\hline \multirow{5}{*}{ B } & 0.15 & 107.11 & 0.21 & 107.40 & 0.12 & 106.86 & 0.22 \\
\hline & 0.6 & 106.90 & 0.23 & 107.20 & 0.12 & 106.86 & 0.22 \\
\hline & 1 & 106.93 & 0.20 & 107.06 & 0.12 & 106.85 & 0.22 \\
\hline & 10 & 106.94 & 0.22 & 107.05 & 0.12 & 105.95 & 0.22 \\
\hline & 30 & 106.75 & 0.22 & 106.54 & 0.12 & 96.40 & 0.23 \\
\hline \multirow{5}{*}{$\mathrm{C} / \mathrm{D}$} & 50 & 90.08 & 0.16 & 89.62 & 0.14 & 90.94 & 0.25 \\
\hline & 120 & 90.04 & 0.17 & 89.20 & 0.14 & 85.85 & 0.25 \\
\hline & 300 & 90.14 & 0.16 & 88.96 & 0.14 & 78.35 & 0.25 \\
\hline & 500 & 90.12 & 0.18 & 88.57 & 0.14 & 73.91 & 0.25 \\
\hline & 1000 & 89.57 & 0.20 & 88.61 & 0.15 & 45.79 & 0.26 \\
\hline
\end{tabular}

Tab. 4 Summary of measured results using different methods. IGUU 2916 Main generator, amplitude setting $60 \mathrm{~dB} \mu \mathrm{V}$. The spectrum amplitude $S(f)$ is given in $\mathrm{dB} \mu \mathrm{V} / \mathrm{MHz}$ and the associated measurement uncertainty in $\mathrm{dB}(\mathrm{k}=2)$.

Method $\mathrm{A}=$ Fourier transform of the time-domain pulse waveform

Method B = Intermediate-frequency measurement method

Method $\mathrm{C}=$ Measurement of pulse amplitude and duration

\section{Acknowledgement}

This work was supported by the project 15RPT01 RFMicrowave. This project has received funding from the EMPIR programme co-financed by the Participating States and from the European Union's Horizon 2020 research and innovation programme.

\section{References}

[1] M. A. Azpúrua, M. Pous, J. A. Oliva, B. Pinter, M. Hudlička and F. Silva, "Waveform Approach for Assessing Conformity of CISPR 16-1-1 Measuring Receivers," IEEE 
Transactions on Instrumentation and Measurement, vol. 67, no. 5, pp. 1187-1198, May 2018.

[2] G. Mahesh, J. T. Vethamoni, M. Vasanthakumar and V. Venkatesan, "Pulse response analysis of quasi-peak detector in EMI receiver," 2015 13th International Conference on Electromagnetic Interference and Compatibility (INCEMIC), Visakhapatnam, 2015, pp. 14-18.

[3] M. A. Azpúrua, M. Pous and F. Silva, "Specifying the Waveforms for the Calibration of CISPR 16-1-1 Measuring Receivers," IEEE Transactions on Electromagnetic Compatibility, 2019 (Early Access) . doi: 10.1109/TEMC.2019.2923813

[4] S. Pasakawee and V. Sittakul, "Calibration and uncertainty evaluation of pulse generator for EMC testing using FFT technique," 2016 13th International Conference on Electrical Engineering/Electronics, Computer, Telecommunications and Information Technology (ECTI-CON), Chiang Mai, 2016, pp. 1-5.

[5] Specification for radio disturbance and immunity measuring apparatus and methods Part 1-1: Radio disturbance and immunity measuring apparatus - Measuring apparatus. EN 55016-1-1 ed. 3 standard, 2014.

[6] CISPR 16-1-1 Ed. 4: Specification for radio disturbance and immunity measuring apparatus and methods - Part 1-1: Radio disturbance and immunity measuring apparatus Measuring apparatus, International Electrotechnical Commission, IEC, 2015.

[7] R. B. Andrews, "An Impulse Spectral Intensity Measurement System," IEEE Transactions on Instrumentation and Measurement, vol. 15, no. 4, pp. 299-303, Dec. 1966.

[8] N. G. Paulter, and D. R. Larson, "Impulse spectrum amplitude uncertainty analysis," Metrologia, vol. 43, no. 6, pp. 477-485, 2006.

[9] Andrews, J. R.; Arthur, M. G.: Spectrum Amplitude - Definition, Generation and Measurement; National Bureau of Standards; 1977

[10] IEEE Standard for the Measurement of Impulse Strength and Impulse Bandwidth; IEEE Std. 376-1975 (inactive - withdrawn)

[11] Instruction Manual for VLF/HF/VHF/UHF Calibration Pulse and Sine Wave Generator IGUU 2916, Schwarzbeck Mess Elektronik, Rev. B [online]. Available: http://schwarzbeck.de/Datenblatt/m2916.pdf

[12] MIL-STD-462, Military Standard: Measurement of Electromagnetic Interference Characteristics, 1967. 
[13] J. R. Palladino, "A New Method for the Spectral Density Calibration of Impulse Generators," IEEE Transactions on Electromagnetic Compatibility, vol. EMC-13, no. 1, pp. 2-7, Feb. 1971.

\section{Biography}

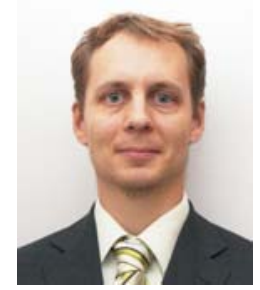

Martin Hudlička (S'04-M'08-SM'15) received the Ing. (M.Sc.) and Ph.D. degrees in electrical engineering from the Czech Technical University, Prague, Czech Republic, in 2004 and 2007, respectively. In 2007, he joined the Department of Primary Metrology of RF Electrical Quantities, Czech Metrology Institute, Prague, where he works as metrologist and researcher. His current research interests include microwave and millimeter-wave measurements and modern communication technologies.

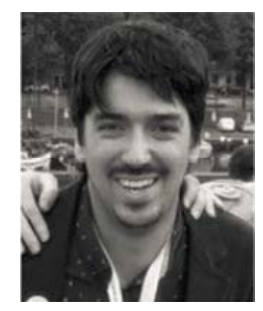

Marco A. Azpúrua (S'07-M'13-SM'-19) received the B.Sc. degree in telecommunications engineering, in 2008, and the M.Sc. degree in electrical engineering from the Universidad Central de Venezuela, Caracas, in 2013. In 2018, received the Ph.D. degree in Electronics Engineering from the Universitat Politècnica de Catalunya (UPC), Barcelona, Spain, for his contributions to Full Time Domain EMI measurements and their applications in complex scenarios. Currently, he is working as Researcher with the Electromagnetic Compatibility Group (GCEM) of the UPC. Formerly, he was a Researcher in the Applied Electromagnetics Laboratory, Instituto de Ingeniería (FII), Caracas, Venezuela. His research interests include electromagnetic compatibility, antenna and microwave measurement technologies, estimation of measurement uncertainty in complex systems and validation methods. 


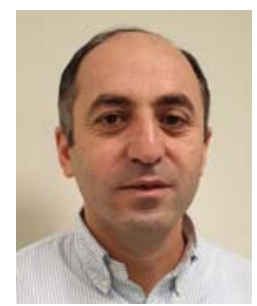

Murat Celep received the technician degree in electronics from the Tekirdag Vocational School, University of Trakya, Edirne, Turkey, in 1992, and the B.Sc., M.Sc., and Ph.D. degrees from the Department of Electronics and Communication Engineering, Kocaeli University, Izmit, Turkey, in 1999, 2004, and 2013, respectively. He joined the RF and Microwave Laboratory, TUBITAK Ulusal Metroloji Enstitüsü (UME), Gebze, Turkey, in 1997. He is involved in performing primary and secondary levels of power, S-parameters, impedance, attenuation, and noise measurements at RF and microwave frequencies, installing and characterizing measurement systems, and uncertainty calculations. 


\title{
Practical aspects of a pulse generator calibration
}

\author{
Martin Hudlička ${ }^{1}$, mhudlicka@cmi.cz, Marco A. Azpúrua ${ }^{2}$, Murat Celep $^{3}$ \\ ${ }^{1}$ Czech metrology institute, Brno, Czech Republic \\ ${ }^{2}$ Universitat Politècnica de Catalunya, Barcelona, Spain \\ ${ }^{3}$ TUBITAK Ulusal Metroloji Enstitüsü (UME), Gebze, Turkey
}

Measuring receivers are used for measurement of radio disturbance in the frequency range typically $9 \mathrm{kHz}$ to $18 \mathrm{GHz}$. Such receivers can be either electromagnetic interference (EMI) receivers or spectrum analyzers with the quasi-peak (QP) detector. Requirements for measuring receivers are discussed in European CISPR documents (Comité International Spécial des Perturbations Rádioélectriques) and US standards ANSI 63.2 (QP parts derived from CISPR). For checking compliance of the receiver with requirements of these standards, pulse generators are needed, which must be calibrated. The calibration of pulse generators is discussed in the standard EN 55016-1-1 [1], which is the harmonized version of the international standard IEC/CISPR 16-1-1 (currently Ed. 4 [2]). In the standard [1], however, only a very brief description of the methods is given and technical details are hidden. The measurement uncertainty of the pulse generator characterization is not discussed. This paper aims to provide a more thorough description of particular calibration methods together with practical hints which may be useful for students, calibration engineers and practitioners.

\section{Pulse generators}

According to [1], a pulse generator is an instrument capable of generating time-domain rectangular pulses, or a pulse-modulated RF signal. Rectangular pulses are typically used for lower frequencies (bands A/B), pulse-modulated RF signals for higher frequencies (bands $\mathrm{C} / \mathrm{D}$ ) because of the risk of receiver damage due to high peak voltages. The base-band pulse generators usually comprise of an energy-storage device (electrostatic, magnetic field) and a switch which discharges a fraction or all of the energy into a load. The pulse-modulated RF generator uses a harmonic signal with a pulse envelope. The spectrum is similar to a rectangular pulse (upconverted to the carrier frequency $f_{c}$ ), maximum of the spectrum is at $f_{c}$. The spectrum is uniform in a given bandwidth, which implies that pulses with longer duration can be used with lower amplitudes compared to base-band pulse generators (lower risk of 
measuring receiver damage). The typical spectrum of a rectangular base-band pulse and a pulse-modulated RF signal is shown in Fig. 1.
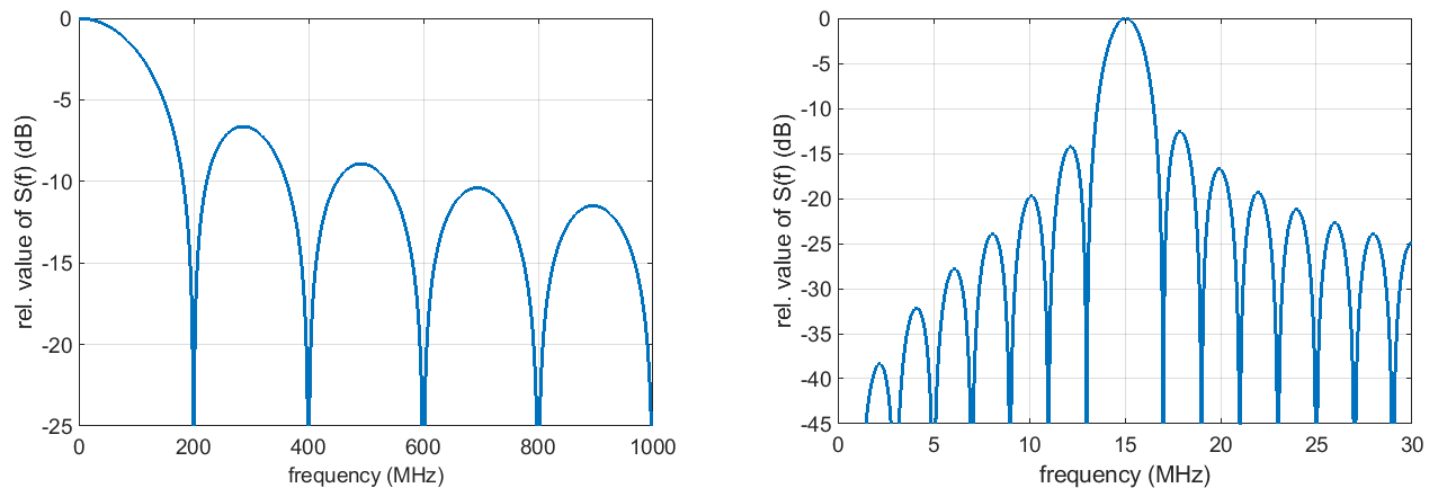

Fig. 1 Typical spectrum of a rectangular base-band pulse (left) and pulse-modulated RF signal (right).

The terminology used with the pulse generator characterization is summarized in Tab. 1 . All quantities for characterization of pulse generators have dimensional units $[\mathrm{V} / \mathrm{Hz}]$ or its mathematical equivalent.

\begin{tabular}{|c|c|c|}
\hline Quantity & Definition & Notes \\
\hline $\begin{array}{l}\text { Spectrum } \\
\text { amplitude }\end{array}$ & $S(f)=2|V(f)|$ & $\begin{array}{l}V(f) \text { is the Fourier transform of the } v(t) \text { signal in } \\
\text { the time-domain }[3] \text {, [4]. It is the measure of the } \\
\text { amplitude spectrum at a certain frequency, } \\
\text { expressed in }[\mathrm{dB} \mu \mathrm{V} / \mathrm{MHz}] \text {. For a rectangular } \\
\text { pulse with amplitude } A \text { and duration } T \text { and low } \\
\text { frequencies }(f<<T) \text { we can write } S(f)=2 A T \text {. }\end{array}$ \\
\hline Impulse area & $A_{i m p}=\int_{0}^{T} v(t) d t$ & $\begin{array}{l}\text { Voltage-time area of a pulse defined by the } \\
\text { integral. Sometimes referred to as impulse } \\
\text { strength, typically expressed in }[\mu \mathrm{Vs}] \text { or } \\
{[\mathrm{dB} \mu \mathrm{Vs}] \text {. Related to spectral density. } T \text { is one }} \\
\text { pulse period. }\end{array}$ \\
\hline Spectral intensity & $S(f)=2 A_{\text {imp }}$ & This definition can be found in [1] and [4]. \\
\hline Spectral density & $D=\sqrt{2} \times 10^{6} A_{i m p}$ & $\begin{array}{l}\text { Defined in }[1] \text {. Alternative term "impulse } \\
\text { strength" can be found. The term } \sqrt{ } 2 \text { stands for } \\
\text { the peak to RMS conversion. } D \text { is calculated in } \\
{[\mu \mathrm{V} / \mathrm{MHz}] \text { for } A_{\text {imp }} \text { given in }[\mu \mathrm{Vs}] \text {. }}\end{array}$ \\
\hline
\end{tabular}

Tab. 1 Quantities used for characterization of pulse generators. 


\section{Calibration methods}

The various pulse generator calibration methods will be demonstrated on the example calibration of the CISPR pulse generator IGUU2916 (base-band pulse generator, manufacturer Schwarzbeck, Germany). The generator is shown in Fig. 2. The methods are finally compared with regards to the measurement uncertainty, feasibility and required instrumentation. The polarity was always $(+)$ and the amplitude of the main generator was always $60 \mathrm{~dB} \mu \mathrm{V}$, the pulse repetition rate was changed according to the band.

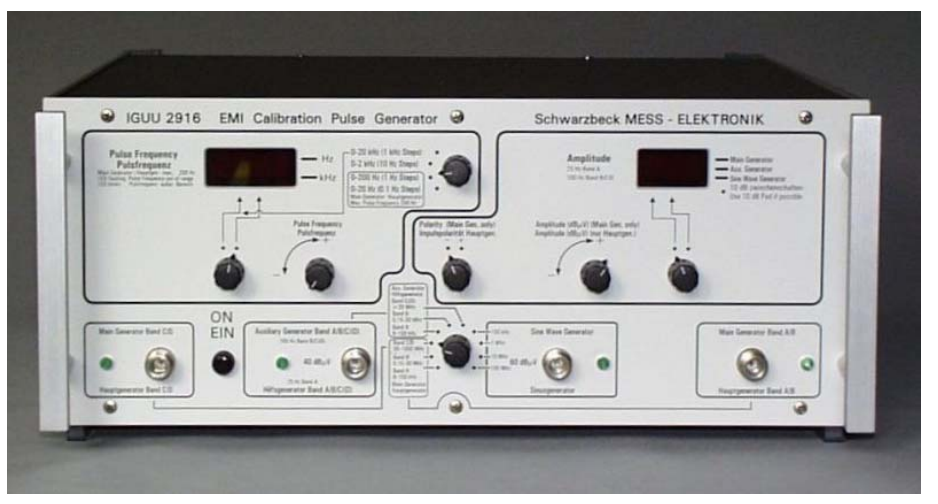

Fig. 2 CISPR pulse generator Schwarzbeck IGUU 2916.

In the EN 55016-1-1 (CISPR 16-1-1) there are specified impulse areas of a typical generator for both open-circuit and $50 \Omega$ load, see Tab. 2. The values shown in this paper correspond to the measurement of the spectrum amplitude (calculated from the impulse area) into $50 \Omega$ nominal load.

\begin{tabular}{|l|c|c|c|c|}
\hline \multicolumn{1}{|c|}{ Band } & A & B & C & D \\
\hline $\begin{array}{l}\text { Frequency } \\
\text { range }\end{array}$ & $\{9-150\} \mathrm{kHz}$ & $\{0.15-30\} \mathrm{MHz}$ & $\{30-300\} \mathrm{MHz}$ & $\{300-1000\} \mathrm{MHz}$ \\
\hline $\begin{array}{l}\text { Impulse area } \\
\text { (open-circuit) }\end{array}$ & $13.5 \mu \mathrm{Vs}$ & $0.316 \mu \mathrm{Vs}$ & $0.044 \mu \mathrm{Vs}$ & $0.044 \mu \mathrm{Vs}$ \\
\hline $\begin{array}{l}\text { Impulse area } \\
\text { (into } 50 \Omega \\
\text { load) }\end{array}$ & $6.75 \mu \mathrm{Vs}$ & $0.158 \mu \mathrm{Vs}$ & $0.022 \mu \mathrm{Vs}$ & $0.022 \mu \mathrm{Vs}$ \\
\hline Repetition rate & $25 \mathrm{~Hz}$ & $100 \mathrm{~Hz}$ & $100 \mathrm{~Hz}$ & $100 \mathrm{~Hz}$ \\
\hline
\end{tabular}

Tab. 2 Impulse area of a generator for different bands specified in the EN 55016-1-1 standard. 


\section{Fourier transform of a time-domain pulse waveform}

The spectrum amplitude is determined by direct acquisition of the pulse generator output voltage using an oscilloscope and conversion into the frequency domain. For this purpose, a digital real-time oscilloscope (DRTO) or an equivalent-time sampling oscilloscope (DSO) can be used. The method is useful for base-band pulse generators, it is simple and time-efficient. Corrections for the cable (attenuator) properties and oscilloscope transfer function must be performed. A DRTO triggers directly the measured pulse. The traceability of DRTO is complicated due to the nonlinear behaviour of modern analogue-to-digital converters and the transfer function correction. A DSO needs an external trigger signal, which is usually derived from the measured signal itself (approx. $20 \mathrm{~ns}$ delay line is used). The measurement is traceable to the electro-optic sampling system. A general diagram of the measurement setup using the DRTO and DSO is shown in Fig. 3.
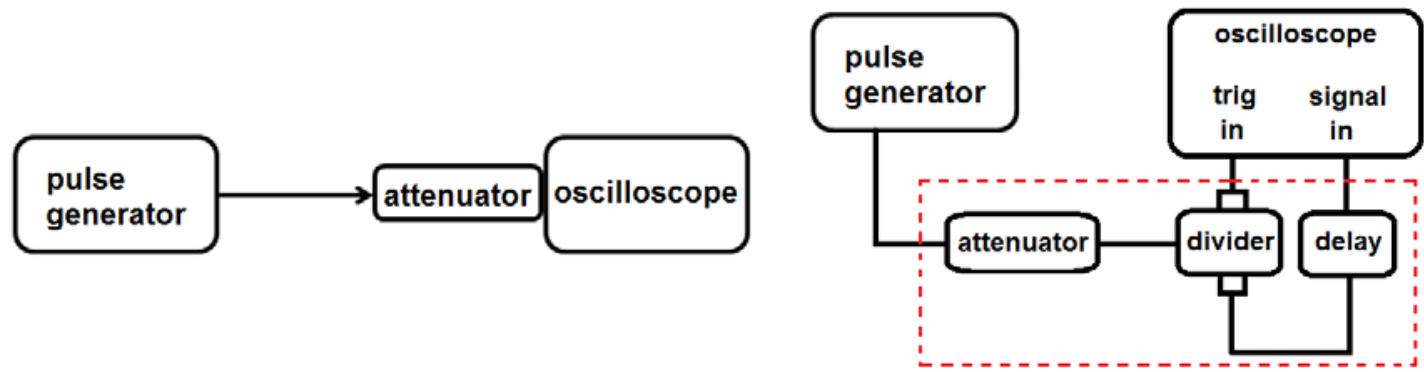

Fig. 3 Typical measurement setup with the use of a DRTO (left) and DSO (right).

The measurement equation is the following:

$$
S(f)=20 \log \left(\frac{|V(f)| \sqrt{2} \cdot 10^{6} \cdot k_{\text {ATT }} \cdot k_{\text {osc }}}{N_{F F T} \Delta f / 10^{6}}\right) \quad[\mathrm{dB} \mu \mathrm{V} / \mathrm{MHz}]
$$

where $V(f)$ is the Fourier transform of the voltage trace from oscilloscope in [V], $N_{F F T}$ is the FFT length, $\Delta f$ is the frequency resolution in $[\mathrm{Hz}], k_{A T T}$ is the total attenuation of the signal path, that is, the cables and external attenuators connected between the generator and oscilloscope, $k_{\text {osc }}$ is a factor taking into account the oscilloscope frequency response. A cable with attenuators on both sides should be used in order to reduce the pulse amplitude and improve the mismatch uncertainty.

Following text shows an example of measured and calculated results. The spectrum amplitude was calculated using (2) from oscilloscope voltage samples corrected for the cable and attenuator and oscilloscope transfer function. Following uncertainty contributions apply: 
- impedance mismatch correction between device 1 and 2 (e.g. between a generator and cable) $u_{M}=20 \log \left(1+\left|\Gamma_{1}\right|\left|\Gamma_{2}\right|\right)(\mathrm{dB})$, where $\Gamma_{1}$ and $\Gamma_{2}$ are linear reflection coefficients of devices 1 and 2, respectively,

- type A uncertainty was calculated from repeated calculations of the spectrum amplitude for all captured time traces, its value was determined from $n$ measurements in a standard way as

$$
u_{A y}=\sqrt{\frac{1}{n(n-1)} \sum_{i=1}^{n}\left(y_{i}-\bar{y}\right)} .
$$

The output reflection coefficient of the IGUU 2916 Main generator is not specified in the datasheet [6] and it was calculated from a hypothetical generator composed of an ideal voltage source with an inner resistance $R_{\text {in }}$ by measuring the output voltage (peak maximum) of the generator into two different load impedances $50 \Omega$ and $1000 \Omega$, respectively.

The measured results of the Fourier transform method with measurement uncertainty are summarized in Tab. 4. The example of measurement uncertainty evaluation for the Band A is given in Tab. 3.

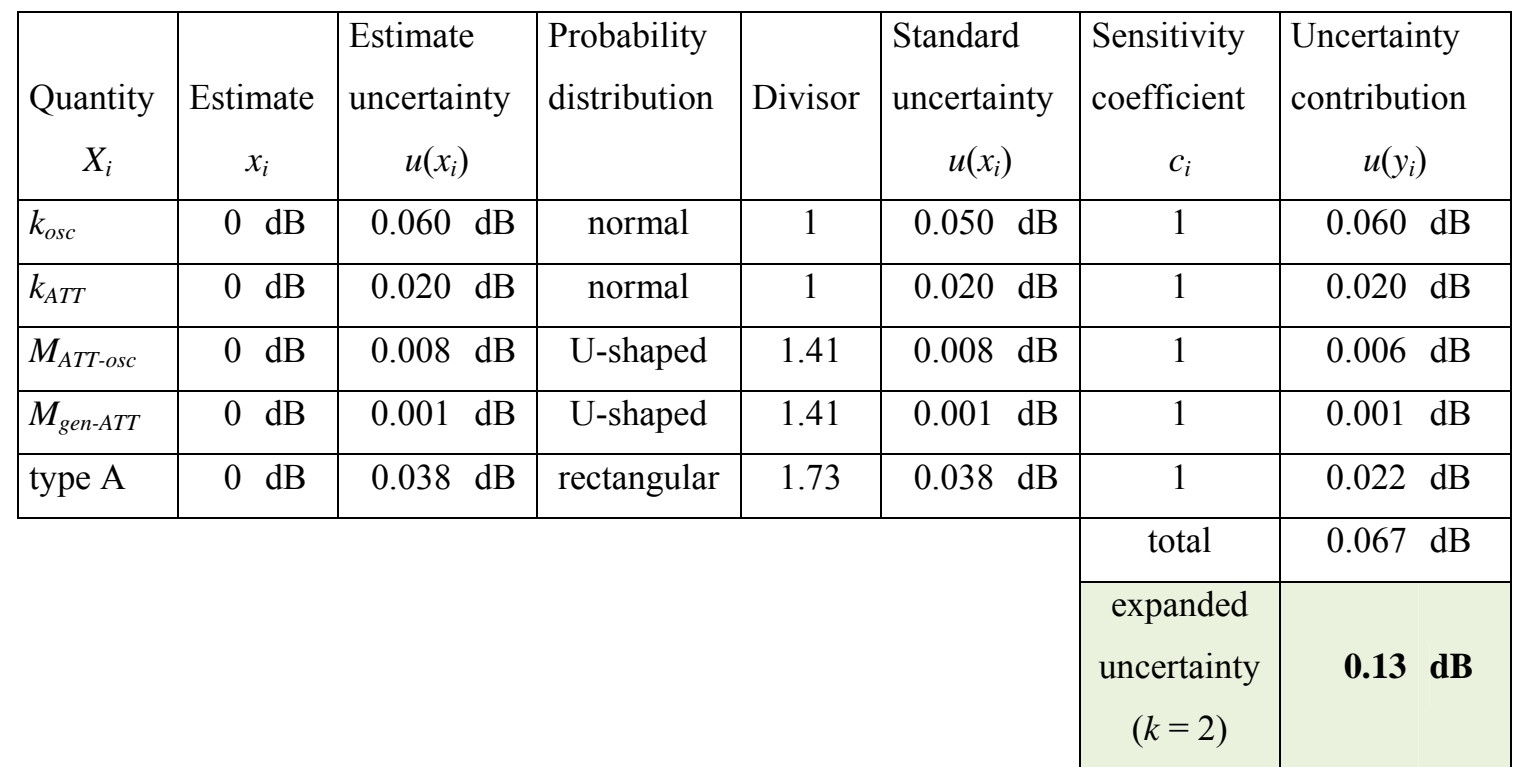

Tab. 3 Example of uncertainty calculation, band $\mathrm{A}, \mathrm{f}_{\mathrm{rep}}=25 \mathrm{~Hz}$, frequency $9 \mathrm{kHz}$.

\section{Intermediate-frequency measurement method}

This method utilizes an EMI measuring receiver and its intermediate frequency output. It is referred to as "video pulse technique" in MIL-STD-462 [5], and it is referred to as "video pulse technique" and "area method" in [1]. The method uses a pulse signal and a reference CW signal (with known level) connected to a narrow-band filter, whereas the output of the 
filter (intermediate frequency) is acquired using an oscilloscope. The spectrum amplitude is then calculated from the response to both input signals at the frequency of the tuned filter (receiver) as follows

$$
S(f)=U_{r m s} / \mathrm{IBW}
$$

where $U_{r m s}(\mathrm{~V})$ is the level of $\mathrm{CW}$ signal which causes equal oscilloscope reading as the pulse signal, IBW $(\mathrm{Hz})$ is the impulse bandwidth of the used filter. The accuracy of the method is dependent on the accurate characterization of the receiver impulse bandwidth IBW. The spectrum amplitude is calculated as the surface under the pulse envelope (i.e. positive amplitudes only), see Fig. 4. The measurement setup is shown in Fig. 5.

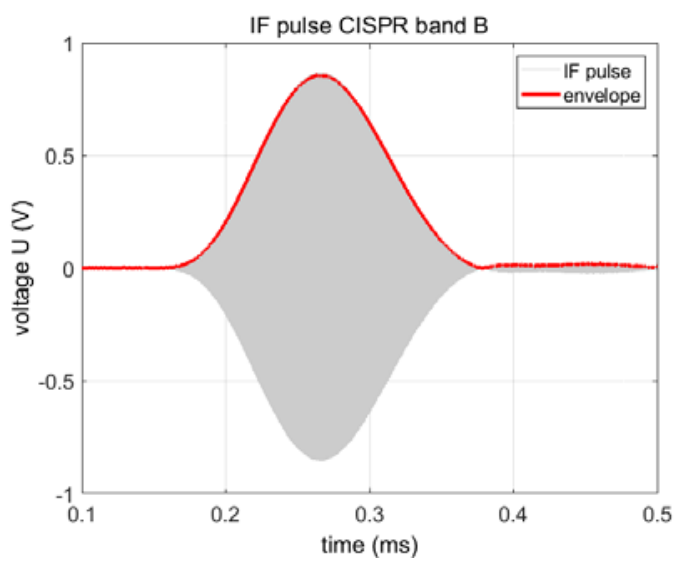

Fig. 4 Intermediate frequency measurement method.

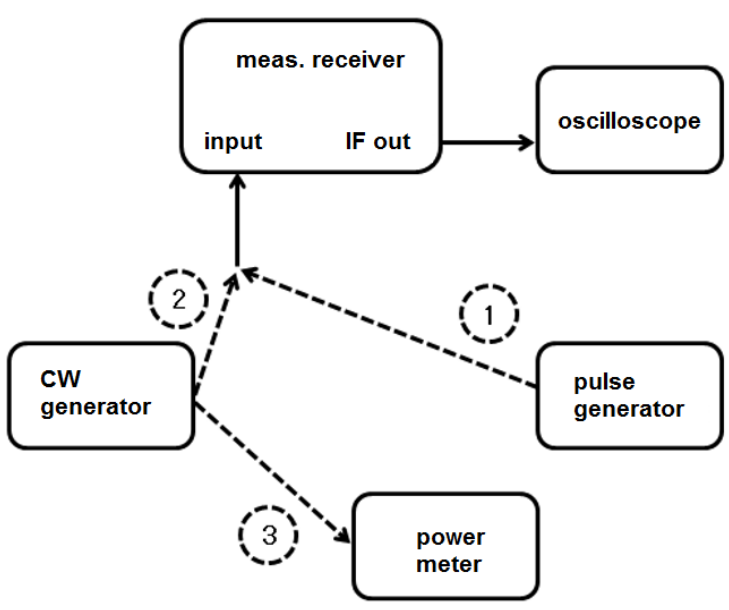

Fig. 5 Measurement setup of the intermediate frequency method. 
The practical procedure is following: in the first step, the pulse generator output is connected to the receiver input and the response of the receiver's IF filter is captured using an oscilloscope (direct connection using a high-grade cable, without attenuators). The peak-topeak amplitude of the trace is measured as well. In the second step, a CW sine signal is connected to the receiver and its amplitude is changed until the oscilloscope peak-to-peak reading is the same as for the pulse signal. The RMS level of this sine signal is measured using a calibrated power meter. The attenuation of the cable from the generator to the receiver and from the receiver IF output to the oscilloscope is not important, as it cancels due to the ratio measurement. The measurement equation is following

$$
S(f)=20 \log \left(\frac{2 V_{p w m, r m s} V_{\text {env }}}{V_{\text {osc }, p p}} k_{\text {peak }} k_{\text {osc }} k_{I B W} I B W \cdot 10^{6}\right) \quad[\mathrm{dB} \mu \mathrm{V} / \mathrm{MHz}]
$$

where $V_{p w m, r m s}$ is the voltage across a $50 \Omega$ load of the CW sine signal calculated from the RMS power measured by the power meter in $[\mu \mathrm{V}]$ $V_{\text {env }}$ is the amplitude of the IF pulse envelope (see the example in Fig. 6) in [V] $V_{\text {osc,pp }}$ is the peak-to-peak amplitude of the receiver response (IF output) to a CW sine signal measured by the oscilloscope in [V]

$I B W$ is the receiver impulse bandwidth in $[\mathrm{Hz}]$ $k_{\text {peak }}$ takes into account the uncertainty of the peak ratio of the response to pulse/CW signal (dimensionless)

$k_{\text {osc }}$ takes into account the oscilloscope frequency response (dimensionless) $k_{I B W}$ takes into account the uncertainty of the determination of the impulse bandwidth (dimensionless).

The receiver impulse bandwidth is calculated as

$$
I B W=\frac{V_{e n v}}{X \cdot \frac{10}{N} \sum_{k=1}^{N} v_{e n v, k}}[\mathrm{~Hz}]
$$

where $V_{e n v}$ is the amplitude of the IF pulse envelope (see see the example in Fig. 6) in [V]

$X$ is the oscilloscope horizontal resolution in [s/div]

$N$ is the number of samples of the envelope [-]

$v_{e n v, k}$ represents the $k$-th sample of the IF pulse envelope in [V]

10 takes into account the number of oscilloscope horizontal screen divisions. 

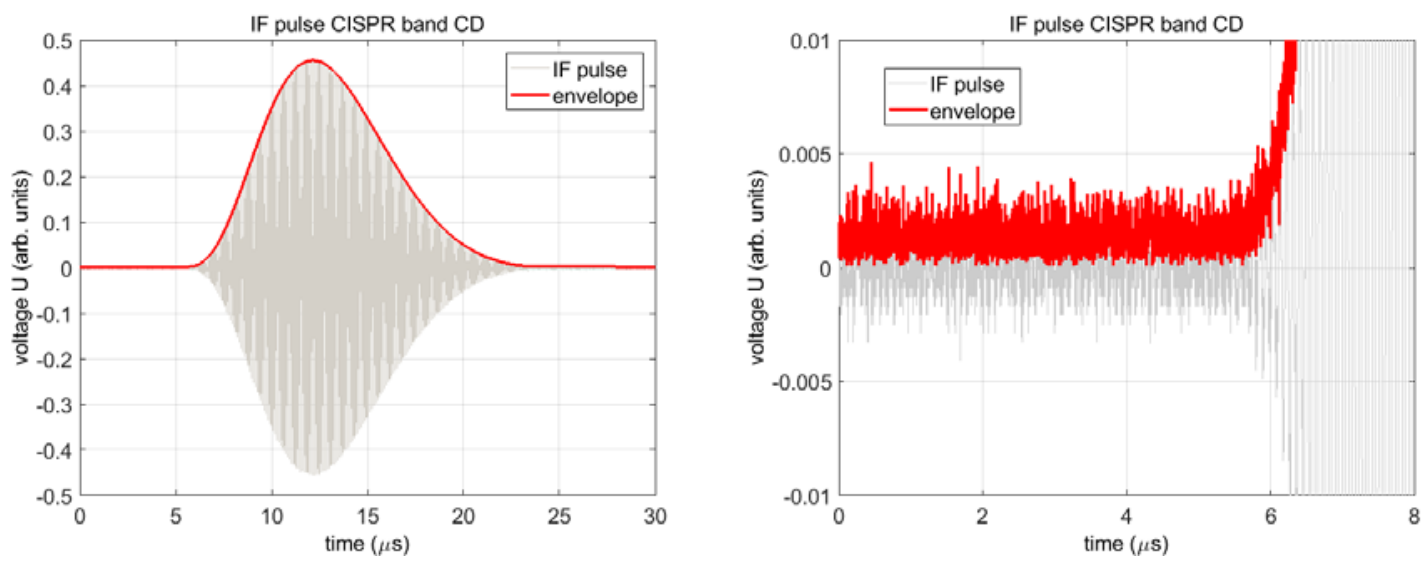

Fig. 6 Response of the receiver to a pulse signal + envelope of the signal (left), unfiltered envelope (right).

The determination of the IF pulse envelope can be done using different methods which results in slightly different calculated receiver impulse bandwidth and consequently spectrum amplitude. Either the envelope can be calculated as a moving average of the voltage IF trace (with e.g. 50 - 200 samples window), or it can be calculated as a magnitude of the Hilbert transform of the IF voltage trace (time-domain). It is convenient to filter the envelope trace using a low-pass filter in order to remove the noise (which is obvious in Fig. 6 right). The area under the envelope is then calculated as a sum of the voltage samples divided by the number of envelope samples. The spectrum amplitude was calculated using (6) from oscilloscope samples of the voltage at the IF output of an EMI receiver and their values together with the measurement uncertainty are given in Tab. 4.

\section{Measurement of pulse amplitude and duration}

This method uses an oscilloscope, whereas the pulse is sampled with high time-resolution. The method is most suitable for pulse-modulated RF generators. The spectrum amplitude is calculated from the area of the pulse $[\mathrm{V} \cdot \mathrm{s}]$. The ideal measurement equation can be written as follows

$$
S(f)=U_{r m s} \cdot T \cdot k_{c o r},
$$

where $U_{r m s}$ is the un-modulated CW signal level, $T$ is the modulation pulse duration and $k_{c o r}$ is a correction factor taking into account the signal level change after switching ON the pulse. The pulse repetition rate should be stable and correction factor should not change with $\mathrm{CW}$ frequency. An example of the pulse modulated $\mathrm{CW}$ signal with frequency $5 \mathrm{MHz}$ is shown in 
Fig. 7 (note the nonzero rising and trailing edge of the pulse due to the modulator nonidealities).

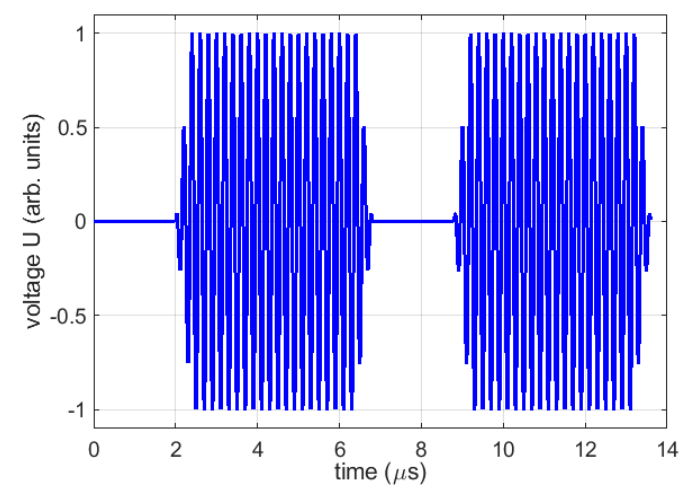

Fig. 7 Measurement of pulse amplitude and duration, pulse-modulated RF generator.

The method is also applicable for base-band pulse generators. The pulse shape must be very close to rectangular, which is not always true for the measured IGUU2916 generator (especially band C/D). The pulse amplitude and duration is measured using an oscilloscope. The pulse shape in the time domain is generally distorted by the transfer function of the cable + attenuator(s) and the oscilloscope transfer function. In order to remove this effect correctly, one has to perform deconvolution of the transfer function in the frequency domain. The pulse shape, however, was acquired using a short oscilloscope epoch (only the pulse visible on the screen), which makes the frequency resolution very coarse and the correction is not easily applicable.

$$
S(f)=20 \log \left(\sqrt{2} A \cdot k_{A T T} \cdot \Delta T \cdot\left|\frac{\sin (\pi f T)}{\pi f T}\right| \cdot 10^{6}\right) \quad[\mathrm{dB} \mu \mathrm{V} / \mathrm{MHz}],
$$

where $A$ is the sum of voltage samples in the pulse trace in $[\mu \mathrm{V}]$,

$\Delta T$ is the time resolution (sampling time) in [s],

$T$ is the total duration of the pulse in [s],

$f$ is the frequency in $[\mathrm{Hz}]$,

$k_{\text {ATT }}$ is the attenuation of the cable + attenuator between the generator and oscilloscope.

Example of the IGUU2916 pulse shape in the C/D band is shown in Fig. 8 for changing the pulse repetition rate. 

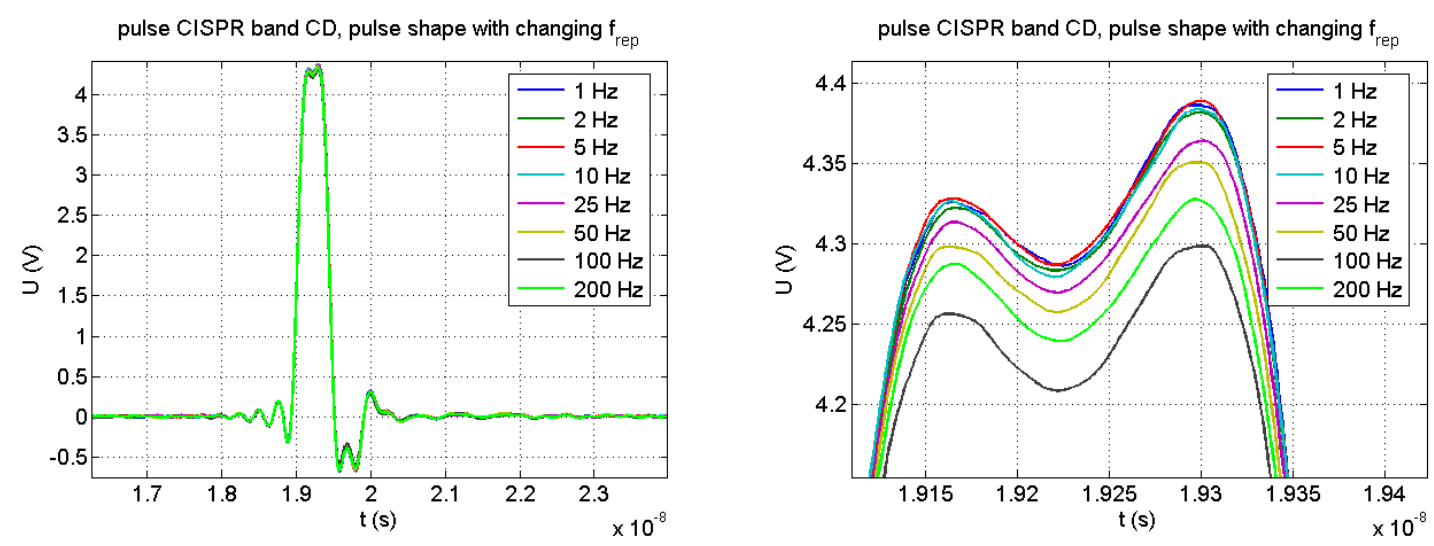

Fig. 8 Pulse shape for the band C/D of the IGUU2916 Main generator with changing pulse repetition rate, detail of the pulse maximum on the right (amplitude without correction for the cable and attenuators).

It can be seen from Fig. 8 the pulse shape in band C/D is distorted and moreover, it contains more noise than in case of the pulses in bands A and B (not shown).

The results are shown in Tab. 4. It can be seen that do not correspond very well with the results of the Fourier transform method or the IF pulse measurement method. The measurement uncertainty mainly comprises of the cable + attenuator correction, impedance mismatch and type A uncertainty. It is obvious that this method is better suitable for pulsemodulated RF generators and not the base-band pulse generators like IGUU 2916.

The reason for the large deviation of some results from the results of other methods may be the presence of noise and undershoots or overshoots in the pulse trace. It can be shown that the contribution of these components to the total surface in $[\mathrm{V} \cdot \mathrm{s}]$ is significant. The signal is noisy and especially in band $\mathrm{C} / \mathrm{D}$, the error can be reduced by summing over only those samples, which correspond to the pulse (the choice of samples may be subjective).

\section{Measurement of one spectrum line amplitude}

The principle of this method is a comparison of one spectrum line of the pulse signal with a known CW signal spectrum (equal frequency). This method assumes the generator pulse repetition frequency is high enough so that only one spectral line falls within the EMI receiver filter bandwidth, see Fig. 9. The nominal filter bandwidths for the $6 \mathrm{~dB}$ amplitude drop are $200 \mathrm{~Hz}$ (band A), $9 \mathrm{kHz}$ (band B) and $120 \mathrm{kHz}$ (band C/D), respectively. This method is referred to as "harmonic measurement" in [1]. 

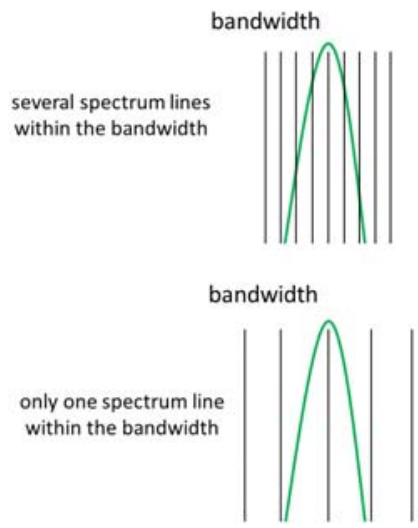
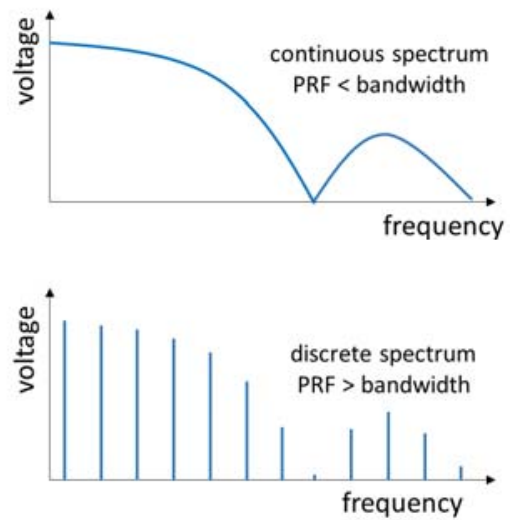

Fig. 9 Measurement of one spectrum line amplitude $(\mathrm{PRF}=$ pulse repetition frequency).

The spectrum amplitude is then calculated using a simple formula

$$
S(f)=\frac{A}{f_{\text {rep }}}
$$

where $A$ is the substitution $\mathrm{CW}$ signal level for the same reading of the receiver and $f_{\text {rep }}$ is the generator pulse repetition frequency. A calibrated measuring receiver is needed in this case. The measurement setup is shown in Fig. 10. The receiver filter should be well symmetrical.

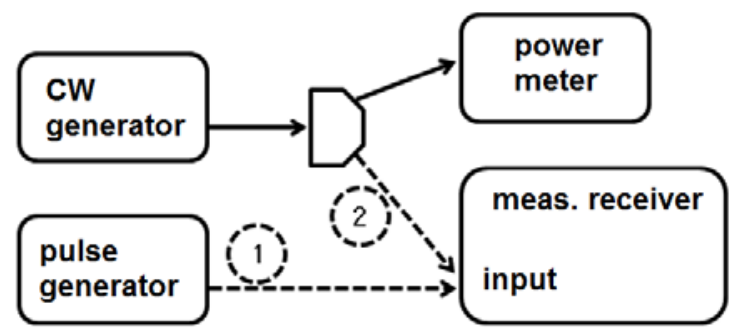

Fig. 10 Measurement setup for the measurement of one spectrum line amplitude.

The maximum pulse repetition rate of the IGUU2916 main generator is $200 \mathrm{~Hz}$, thus only the band A could be possible to verify. In other bands, the pulse repetition frequency would have to be sufficient. Results of this method are not compared with the other methods in Tab. 4.

\section{Conclusion}

In this guide, various methods for calibration of pulse generators are discussed and results of measurement of the spectrum amplitude of a particular pulse generator Schwarzbeck IGUU 2916 are presented. The spectrum amplitude was evaluated using following methods: (a) 
Fourier transform of the time-domain pulse waveform; (b) intermediate-frequency measurement method; (c) measurement of pulse amplitude and duration and (d) measurement of one spectrum line amplitude. The method (a) can achieve the lowest measurement uncertainty and is simple to perform. It requires fast digital real-time or sampling oscilloscope and the spectrum of the measured waveform must be corrected for known attenuation of the signal path. The method (b) uses an EMI receiver which is more common in calibration laboratories. The measurement uncertainty is comparable to method (a). One has to be careful and not to overload the receiver input, as time-domain pulse generators use high peak amplitudes which may destroy the input mixer. Method (c) is more suitable for pulsemodulated RF generators and the results for a base-band pulse generator IGUU 2916 band $\mathrm{C} / \mathrm{D}$ are not reliable due to the distorted pulse shape. The measurement uncertainty is slightly higher than that of methods (a), (b). Method (d) is applicable only for pulse generators with very high pulse repetition rates (at least $500 \mathrm{~Hz}$ for band A, at least $10 \mathrm{kHz}$ for band $\mathrm{B}$ and at least $120 \mathrm{kHz}$ for band C/D). The results achieved for band A and B of the IGUU 2916 Aux generator are comparable to method (a) for the same generator, however, the measurement uncertainty is rather high due to noise and low repeatability of the pulses. The goal uncertainty of maximum $\pm 0.5 \mathrm{~dB}$ given in standards could not be achieved using method (d). The measured results of methods (a) to (c) with measurement uncertainties are summarized in Tab. 4. 


\begin{tabular}{|c|c|c|c|c|c|c|c|}
\hline \multirow[b]{2}{*}{ Band } & \multirow[b]{2}{*}{$\begin{array}{c}\text { Frequency } \\
(\mathrm{MHz})\end{array}$} & \multicolumn{2}{|c|}{ Method A } & \multicolumn{2}{|c|}{ Method B } & \multicolumn{2}{|c|}{ Method C } \\
\hline & & $S(f)$ & Unc. & $S(f)$ & Unc. & $S(f)$ & Unc. \\
\hline \multirow{5}{*}{ A } & 0.009 & 139.90 & 0.13 & 139.84 & 0.21 & 139.81 & 0.22 \\
\hline & 0.01 & 139.83 & 0.13 & 139.87 & 0.21 & 139.81 & 0.22 \\
\hline & 0.05 & 139.77 & 0.12 & 139.83 & 0.21 & 139.68 & 0.22 \\
\hline & 0.1 & 139.79 & 0.11 & 139.84 & 0.21 & 139.24 & 0.22 \\
\hline & 0.15 & 139.79 & 0.11 & 140.25 & 0.21 & 138.49 & 0.22 \\
\hline \multirow{5}{*}{ B } & 0.15 & 107.11 & 0.21 & 107.40 & 0.12 & 106.86 & 0.22 \\
\hline & 0.6 & 106.90 & 0.23 & 107.20 & 0.12 & 106.86 & 0.22 \\
\hline & 1 & 106.93 & 0.20 & 107.06 & 0.12 & 106.85 & 0.22 \\
\hline & 10 & 106.94 & 0.22 & 107.05 & 0.12 & 105.95 & 0.22 \\
\hline & 30 & 106.75 & 0.22 & 106.54 & 0.12 & 96.40 & 0.23 \\
\hline \multirow{5}{*}{$\mathrm{C} / \mathrm{D}$} & 50 & 90.08 & 0.16 & 89.62 & 0.14 & 90.94 & 0.25 \\
\hline & 120 & 90.04 & 0.17 & 89.20 & 0.14 & 85.85 & 0.25 \\
\hline & 300 & 90.14 & 0.16 & 88.96 & 0.14 & 78.35 & 0.25 \\
\hline & 500 & 90.12 & 0.18 & 88.57 & 0.14 & 73.91 & 0.25 \\
\hline & 1000 & 89.57 & 0.20 & 88.61 & 0.15 & 45.79 & 0.26 \\
\hline
\end{tabular}

Tab. 4 Summary of measured results using different methods. IGUU 2916 Main generator, amplitude setting $60 \mathrm{~dB} \mu \mathrm{V}$. The spectrum amplitude $S(f)$ is given in $\mathrm{dB} \mu \mathrm{V} / \mathrm{MHz}$ and the associated measurement uncertainty in $\mathrm{dB}(\mathrm{k}=2)$.

Method $\mathrm{A}=$ Fourier transform of the time-domain pulse waveform

Method B = Intermediate-frequency measurement method

Method $\mathrm{C}=$ Measurement of pulse amplitude and duration

\section{Acknowledgement}

This work was supported by the project 15RPT01 RFMicrowave. This project has received funding from the EMPIR programme co-financed by the Participating States and from the European Union's Horizon 2020 research and innovation programme. 


\section{References}

[1] Specification for radio disturbance and immunity measuring apparatus and methods Part 1-1: Radio disturbance and immunity measuring apparatus - Measuring apparatus. EN 55016-1-1 ed. 3 standard, 2014.

[2] CISPR 16-1-1 Ed. 4: Specification for radio disturbance and immunity measuring apparatus and methods - Part 1-1: Radio disturbance and immunity measuring apparatus Measuring apparatus, International Electrotechnical Commission, IEC, 2015.

[3] Andrews, J. R.; Arthur, M. G.: Spectrum Amplitude - Definition, Generation and Measurement; National Bureau of Standards; 1977

[4] IEEE Standard for the Measurement of Impulse Strength and Impulse Bandwidth; IEEE Std. 376-1975 (inactive - withdrawn)

[5] MIL-STD-462, Military Standard: Measurement of Electromagnetic Interference Characteristics, 1967.

[6] Instruction Manual for VLF/HF/VHF/UHF Calibration Pulse and Sine Wave Generator IGUU 2916, Schwarzbeck Mess Elektronik, Rev. B [online]. Available: http://schwarzbeck.de/Datenblatt/m2916.pdf

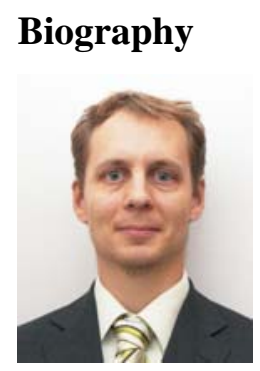

Martin Hudlička (S'04-M'08-SM'15) received the Ing. (M.Sc.) and Ph.D. degrees in electrical engineering from the Czech Technical University, Prague, Czech Republic, in 2004 and 2007, respectively. In 2007, he joined the Department of Primary Metrology of RF Electrical Quantities, Czech Metrology Institute, Prague, where he works as metrologist and researcher. His current research interests include microwave and millimeter-wave measurements and modern communication technologies. 


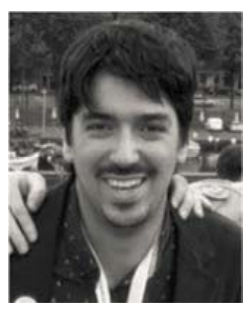

Marco A. Azpúrua (S'07-M'13-SM'-19) received the B.Sc. degree in telecommunications engineering, in 2008, and the M.Sc. degree in electrical engineering from the Universidad Central de Venezuela, Caracas, in 2013. In 2018, received the Ph.D. degree in Electronics Engineering from the Universitat Politècnica de Catalunya (UPC), Barcelona, Spain, for his contributions to Full Time Domain EMI measurements and their applications in complex scenarios. Currently, he is working as Researcher with the Electromagnetic Compatibility Group (GCEM) of the UPC. Formerly, he was a Researcher in the Applied Electromagnetics Laboratory, Instituto de Ingeniería (FII), Caracas, Venezuela. His research interests include electromagnetic compatibility, antenna and microwave measurement technologies, estimation of measurement uncertainty in complex systems and validation methods.

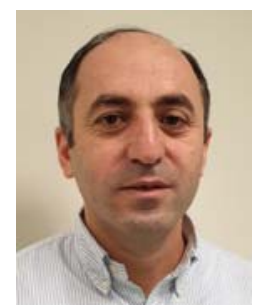

Murat Celep received the technician degree in electronics from the Tekirdag Vocational School, University of Trakya, Edirne, Turkey, in 1992, and the B.Sc., M.Sc., and Ph.D. degrees from the Department of Electronics and Communication Engineering, Kocaeli University, Izmit, Turkey, in 1999, 2004, and 2013, respectively. He joined the RF and Microwave Laboratory, TUBITAK Ulusal Metroloji Enstitüsü (UME), Gebze, Turkey, in 1997. He is involved in performing primary and secondary levels of power, S-parameters, impedance, attenuation, and noise measurements at RF and microwave frequencies, installing and characterizing measurement systems, and uncertainty calculations. 